DOI 10.4171/JEMS/218

Boris Adamczewski · Yann Bugeaud

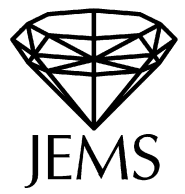

\title{
Transcendence measures for continued fractions involving repetitive or symmetric patterns
}

Received February 13, 2008 and in revised form May 16, 2008

\begin{abstract}
There is a long tradition in constructing explicit classes of transcendental continued fractions and especially transcendental continued fractions with bounded partial quotients. By means of the Schmidt Subspace Theorem, existing results were recently substantially improved by the authors in a series of papers, providing new classes of transcendental continued fractions. It is the purpose of the present work to show how the Quantitative Subspace Theorem yields transcendence measures for (most of) these numbers.
\end{abstract}

\section{Introduction}

It was observed long ago (see e.g., [33] or [21, p. 62]) that Roth's theorem [29] and its $p$-adic extension established by Ridout [28] can be used to prove the transcendence of real numbers whose expansion in some integer base contains repetitive patterns. This was properly written only in 1997, by Ferenczi and Mauduit [22], who adopted a point of view of combinatorics on words before applying the above mentioned theorems from Diophantine approximation to establish e.g., the transcendence of numbers with a low complexity expansion. Their combinatorial transcendence criterion was subsequently considerably improved in [10] by means of the multidimensional extension of Roth's theorem established by W. M. Schmidt, commonly referred to as the Schmidt Subspace Theorem [30, 31]. As shown in [4], this powerful criterion has many applications and yields among other things the transcendence of irrational real numbers whose expansion in some integer base can be generated by a finite automaton. The latter result was generalized in [8], where we gave transcendence measures for a large class of real numbers shown to be transcendental in [4]. The key ingredient for the proof is then the Quantitative Subspace Theorem [32]. We described in [7] a general method that allows us in principle to get transcendence measures for real numbers that are proved to be transcendental by an application of Roth's or Schmidt's theorem, or one of their extensions.

B. Adamczewski: CNRS, Université de Lyon, Université Lyon 1, Institut Camille Jordan, Bât. Braconnier, 21 avenue Claude Bernard, 69622 Villeurbanne Cedex, France; e-mail: Boris.Adamczewski@math.univ-lyon1.fr

Y. Bugeaud: Université de Strasbourg, U. F. R. de mathématiques, 7, rue René Descartes, 67084 Strasbourg Cedex, France; e-mail: bugeaud@math.unistra.fr

Mathematics Subject Classification (2010): 11J82 
Besides expansions in integer bases, a classical way to represent a real number is by its continued fraction expansion. There is actually a long tradition in constructing explicit classes of transcendental continued fractions and especially transcendental continued fractions with bounded partial quotients [25, 14, 27, 12]. Again by means of the Schmidt Subspace Theorem, existing results were recently substantially improved in a series of papers [1, 5, 6, 9, 18], providing new classes of transcendental continued fractions. It is the purpose of the present work to show how the Quantitative Subspace Theorem yields transcendence measures for (most of) these numbers, following the approach initiated in [7]. These measures allow us to locate such numbers in the classification of real numbers defined in 1932 by Mahler [24] and recalled below.

For every integer $d \geq 1$ and every real number $\xi$, we denote by $w_{d}(\xi)$ the supremum of the exponents $w$ for which

$$
0<|P(\xi)|<H(P)^{-w}
$$

has infinitely many solutions in integer polynomials $P(X)$ of degree at most $d$. Here, $H(P)$ stands for the naïve height of the polynomial $P(X)$, that is, the maximum of the absolute values of its coefficients. Further, we set $w(\xi)=\lim _{\sup _{d \rightarrow \infty}}\left(w_{d}(\xi) / d\right)$ and, according to Mahler [24], we say that $\xi$ is an

- A-number if $w(\xi)=0$;

- $S$-number if $0<w(\xi)<\infty$;

- $T$-number if $w(\xi)=\infty$ and $w_{d}(\xi)<\infty$ for any integer $d \geq 1$;

- $U$-number if $w(\xi)=\infty$ and $w_{d}(\xi)=\infty$ for some integer $d \geq 1$.

An important feature of this classification is that two transcendental real numbers that belong to different classes are algebraically independent. The $A$-numbers are precisely the algebraic numbers and, in the sense of the Lebesgue measure, almost all numbers are $S$-numbers. The existence of $T$-numbers remained an open problem during nearly forty years, until it was confirmed by Schmidt (see Chapter 3 of [17] for references and further results). The set of $U$-numbers can be further divided into countably many subclasses according to the value of the smallest integer $d$ for which $w_{d}(\xi)$ is infinite.

Definition 1.1. Let $\ell \geq 1$ be an integer. A real number $\xi$ is a $U_{\ell}$-number if $w_{\ell}(\xi)$ is infinite and $w_{d}(\xi)$ is finite for $d=1, \ldots, \ell-1$.

The Liouville numbers are precisely the $U_{1}$-numbers.

To give a flavour of the results proved in the present paper, we quote below a theorem established in 1962 by A. Baker [14].

Theorem (A. Baker). Consider a quasi-periodic continued fraction

$$
\xi=[a_{0}, a_{1}, \ldots, a_{n_{0}-1}, \underbrace{a_{n_{0}}, \ldots, a_{n_{0}+r_{0}-1}}_{\lambda_{0} \text { times }}, \underbrace{a_{n_{1}}, \ldots, a_{n_{1}+r_{1}-1}}_{\lambda_{1} \text { times }}, \ldots],
$$

where the notation implies that $n_{k}=n_{k-1}+\lambda_{k-1} r_{k-1}$ and the $\lambda_{k}$ 's indicate the number of times a block of partial quotients is repeated. Suppose that the sequences $\left(a_{n}\right)_{n \geq 0}$ and $\left(r_{n}\right)_{n \geq 0}$ are respectively bounded by $A$ and $K$. Set

$$
L=\limsup _{k \rightarrow \infty} \lambda_{k} / \lambda_{k-1}, \quad \ell=\liminf _{k \rightarrow \infty} \lambda_{k} / \lambda_{k-1} .
$$


If $L$ is infinite and $\ell>1$, then $\xi$ is a $U_{2}$-number. Furthermore, if $L$ is finite and $\ell>$ $\exp \left(4 A^{K}\right)$, then $\xi$ is either an $S$-number or a $T$-number.

Baker's theorem shows that the above quasi-periodic continued fractions for which $\ell$ is sufficiently large cannot include $U_{d}$-numbers with $d \geq 3$, that is, there is a gap in the type of transcendental numbers given by them. To the best of our knowledge, this remains up to now the only result in the literature providing a transcendence measure for transcendental numbers defined via their continued fraction expansion as in [25, 14, 27, 12, 1, 5, 6, 9, 18].

The approach of the present paper leads to an improvement of Baker's result. In Theorem 3.2 below, we obtain the same conclusion as in the Theorem above, with the assumption $\ell>\exp \left(4 A^{K}\right)$ replaced by the much weaker one $\ell>1$. In particular, our result does not depend on the values of $A$ or $K$. The key point in the study of the continued fractions considered by Baker is that some blocks of partial quotients are repeated consecutively an arbitrarily large number of times. Besides these quasi-periodic continued fractions, our method also applies to continued fractions involving repetitive patterns in a more hidden form, as well as symmetric patterns. An emblematic example is given by the Thue-Morse continued fraction

$$
\xi_{\mathbf{t}}=[1,2,2,1,2,1,1,2, \ldots],
$$

whose sequence of partial quotients is the Thue-Morse infinite word on the alphabet $\{1,2\}$, that is, the $n$-th partial quotient of $\xi$ is equal to 1 if the sum of the binary digits of $n$ is even and it is equal to 2 otherwise. Although the Thue-Morse sequence is repetitive in the sense of Theorem 2.1 below, it is well-known that it does not contain a finite word repeated consecutively more than twice. The fact that $\xi_{\mathbf{t}}$ is transcendental was established by M. Queffélec [27] only in 1998. As a consequence of Theorems 2.1 and 4.1, we strengthen her result by proving that $\xi_{\mathrm{t}}$ is either an $S$-number or a $T$-number. This apparently provides the first transcendence measures for real numbers of this shape.

All along this paper, we study continued fractions

$$
\xi:=\left[a_{0}, a_{1}, \ldots, a_{\ell}, \ldots\right]
$$

such that the sequence $\left(q_{\ell}^{1 / \ell}\right)_{\ell \geq 1}$ is bounded. Here, $q_{\ell}$ stands for the denominator of the $\ell$-th convergent to $\xi$. We recall that this condition is in general very easy to check, and is not very restrictive, since it is satisfied by almost all real numbers (with respect to the Lebesgue measure). It is in particular always the case when the sequence $\left(a_{\ell}\right)_{\ell \geq 0}$ is bounded.

Our paper is organized as follows. In Section 2, we state a general result, Theorem 2.1 , providing transcendence measures for a large class of continued fractions. These are termed purely stammering continued fractions. Following [1], we then illustrate this result with some applications to various well-known classes of transcendental continued fractions including those arising from Sturmian, morphic and linearly recurrent infinite words. In Section 3, we improve, in the spirit of [5], the theorem of Baker previously mentioned. Transcendence measures for continued fraction involving symmetric patterns are then discussed in Section 4. We gather some auxiliary results in Section 5 and recall in Section 6 our main tool, the Quantitative Subspace Theorem. The proofs of our results are postponed to Sections 7, 8, 9 and 10. 


\section{Transcendence measures for purely stammering continued fractions}

Throughout the present text, we adopt the point of view from combinatorics on words. Let $\mathcal{A}$ be a given set, not necessarily finite. The length of a word $W$ on the alphabet $\mathcal{A}$, that is, the number of letters composing $W$, is denoted by $|W|$. For any positive integer $\ell$, we write $W^{\ell}$ for the word $W \ldots W$ ( $\ell$ times repeated concatenation of $W$ ). We denote by $W^{\infty}$ the infinite word obtained by concatenation of infinitely many copies of $W$. For any positive real number $x$, we denote by $W^{x}$ the word $W^{\lfloor x\rfloor} W^{\prime}$, where $W^{\prime}$ is the prefix of $W$ of length $\lceil(x-\lfloor x\rfloor)|W|\rceil$. Here, and in what follows, $\lfloor y\rfloor$ and $\lceil y\rceil$ denote, respectively, the integer part and the upper integer part of the real number $y$. The study of repetitive patterns occurring in finite or infinite words is a classical topic from combinatorics on words. Several exponents have been introduced to measure the presence of such patterns. Among them, the initial critical exponent of an infinite word $\mathbf{a}=\left(a_{n}\right)_{n \geq 0}$, denoted by ice(a), is defined as the supremum of the real numbers $x$ for which there are arbitrarily long prefixes of $\mathbf{a}$ of the form $W^{x}$. Clearly, ice(a) is always at least equal to 1 and is infinite if $\mathbf{a}$ is a purely periodic sequence. This exponent was introduced in [16] for reasons apparently unrelated to transcendence.

As we will see, the initial critical exponent plays an important role in our context. It essentially allows us to distinguish among the large class of continued fractions considered in Theorem 2.1 the $U_{2}$-numbers from the $S$ - or $T$-numbers. In particular, we show that to decide whether such a continued fraction $\xi$ is a $U_{2}$-number reduces to a purely combinatorial question about the infinite word formed by the partial quotients of $\xi$.

\subsection{Main results}

Let $w>1$ be a real number. We say that a sequence $\mathbf{a}=\left(a_{\ell}\right)_{\ell \geq 0}$ satisfies Condition $(*)_{\widehat{w}}$ if $\mathbf{a}$ is not eventually periodic and if there exists an infinite sequence of finite words $\left(V_{n}\right)_{n \geq 1}$ such that:

(i) for every integer $n \geq 1$, the word $V_{n}^{w}$ is a prefix of the word a;

(ii) the sequence $\left(\left|V_{n}\right|\right)_{n \geq 1}$ is (strictly) increasing;

(iii) the sequence $\left(\left|V_{n+1}\right| /\left|V_{n}\right|\right)_{n \geq 1}$ is bounded.

We establish the following result.

Theorem 2.1. Let $\mathbf{a}=\left(a_{\ell}\right)_{\ell \geq 0}$ be a sequence of positive integers satisfying Condition $(*)_{\widehat{w}}$ for some $w>1$. Let $\left(p_{\ell} / q_{\ell}\right)_{\ell \geq 1}$ be the sequence of convergents to the real number

$$
\xi:=\left[a_{0}, a_{1}, \ldots, a_{\ell}, \ldots\right]
$$

and assume that the sequence $\left(q_{\ell}^{1 / \ell}\right)_{\ell \geq 1}$ is bounded. If ice $(\mathbf{a})$ is finite, then $\xi$ is either an $S$-number or a $T$-number; otherwise, $\xi$ is a $U_{2}$-number. Moreover, if ice(a) is finite, then there exists a constant $c$ independent of $d$ such that

$$
w_{d}(\xi) \leq \exp \left(c(\log 3 d)^{3}(\log \log 3 d)^{2}\right) \quad(d \geq 1) .
$$


In the case where $w \geq 2$ in Theorem 2.1, we are actually able to derive a better transcendence measure, namely to replace (2.1) by

$$
w_{d}(\xi) \leq \exp \left(c(\log 3 d)^{2}(\log \log 3 d)\right), \quad(d \geq 1) .
$$

The fact that the real number $\xi$ is transcendental when the sequence a only satisfies assumptions (i) and (ii) of Condition $(*) \widehat{w}$ is the main result of [1]. In the remainder of this section, we point out several consequences of Theorem 2.1 for various classes of continued fractions already considered in the literature and in particular in [1]. The interested reader will find in that paper more explanations motivating the study of these numbers.

\subsection{Applications to Sturmian, morphic, and linearly recurrent continued fractions}

Let $\alpha>1$ be an irrational real number and $\rho$ be a positive real number. A sequence $\left(a_{\ell}\right)_{\ell \geq 0}$ on the alphabet $\{a, b\}$ is a Sturmian sequence of slope $\alpha$ and intercept $\rho$ if either

$$
a_{\ell}=\lfloor(\ell+1) \alpha+\rho\rfloor-\lfloor\ell \alpha+\rho\rfloor \quad(\ell \geq 0),
$$

or

$$
a_{\ell}=\lceil(\ell+1) \alpha+\rho\rceil-\lceil\ell \alpha+\rho\rceil \quad(\ell \geq 0) .
$$

Such sequences only take two distinct values which are positive integers. For convenience, we will only consider Sturmian sequences defined as in (2.3), so that $\alpha$ and $\rho$ determine a unique Sturmian sequence. The real number $\xi_{\alpha, \rho}:=\left[a_{0}, a_{1}, a_{2}, \ldots\right]$ is then termed a Sturmian continued fraction. The main result of [12] is that Sturmian continued fractions are all transcendental. We make this result more precise.

Theorem 2.2.1. Let $\alpha>1$ be an irrational real number and $\rho$ be a positive real number. Let $\mathbf{a}=\left(a_{\ell}\right)_{\ell \geq 0}$ be a Sturmian sequence of slope $\alpha$ and intercept $\rho$. Then the real number

$$
\xi_{\alpha, \rho}:=\left[a_{0}, a_{1}, \ldots, a_{\ell}, \ldots\right]
$$

is a $U_{2}$-number if and only if $\alpha$ has unbounded partial quotients. If $\alpha$ has bounded partial quotients, then $\xi_{\alpha, \rho}$ satisfies the transcendence measure given in (2.1) and is thus either an $S$-number or a $T$-number.

It follows from Theorem 2.2.1 that $\xi_{\sqrt{2}, e}$ is either an $S$-number or a $T$-number, while $\xi_{e, \sqrt{2}}$ is a $U_{2}$-number. By one of the properties of Mahler's classification recalled in Section 1, these two real numbers are algebraically independent. The proof of Theorem 2.2.1 is postponed to Section 10.

We turn now to an application of Theorem 2.1 to real numbers whose continued fraction expansion can be fixed by a morphism of a free monoid. Given a finite set $\mathcal{A}$, we denote by $\mathcal{A}^{*}$ the free monoid generated by $\mathcal{A}$. The empty word stands for the neutral element of $\mathcal{A}^{*}$. A map from $\mathcal{A}$ into $\mathcal{A}^{*}$ can be extended to an endomorphism of the free monoid $\mathcal{A}^{*}$, which itself can be extended by continuity to a map from $\mathcal{A}^{*} \cup \mathcal{A}^{\mathbb{N}}$ (endowed with its natural topology) into itself. We call such a map a morphism, so that a morphism 
is now allowed to act on infinite words as well. An infinite word $\mathbf{a}$ is a non-trivial fixed point of a morphism $\sigma$ if there exists a letter $a$ in $\mathcal{A}$ such that $\mathbf{a}=\lim _{n \rightarrow \infty} \sigma^{n}(a)$ and a is not eventually periodic. Such an infinite word is said to be recurrent if its first letter occurs at least twice.

Theorem 2.2.2. Let $\sigma$ be a morphism defined over a finite set of positive integers. Let $\mathbf{a}=\left(a_{\ell}\right)_{\ell \geq 0}$ be an infinite word which is a non-trivial recurrent fixed point of $\sigma$ and set $\xi:=\left[a_{0}, a_{1}, \ldots, a_{\ell}, \ldots\right]$. Then $\xi$ is a transcendental number satisfying the transcendence measure given in (2.1) and is thus either an $S$-number or a T-number.

In Section 8, we explain how Theorem 2.2.2 can be deduced from Theorem 2.1. As noticed in [1] (see the proof of Corollary 2), a non-trivial fixed point of a binary morphism is recurrent. We thus deduce from Theorem 2.2.2 the following result.

Corollary 2.2.3. Let $a$ and $b$ be two distinct positive integers. Let $\sigma$ be a binary morphism defined over $\{a, b\}$. Let $\mathbf{a}=\left(a_{\ell}\right)_{\ell \geq 0}$ be a non-trivial fixed point for $\sigma$ and set $\xi:=\left[a_{0}, a_{1}, \ldots, a_{\ell}, \ldots\right]$. Then $\xi$ is a transcendental number satisfying the transcendence measure given in (2.1) and is thus either an $S$-number or a $T$-number.

We end this section with an application of Theorem 2.1 to linearly recurrent continued fractions. Given a positive integer $k$, an infinite word $\mathbf{a}$ is said to be $k$-linearly recurrent if for every positive integer $n$ each word of length $n$ occurring in a has at least one occurrence in every factor of a whose length exceeds $\mathrm{kn}$. A sequence is said to be linearly recurrent if it is $k$-linearly recurrent for some positive integer $k$. A number of famous sequences in combinatorics on words and symbolic dynamics turn out to share this property. The proof of Theorem 5 of [1] shows that an aperiodic linearly recurrent sequence satisfies Condition $(*) \widehat{w}$ for some real number $w>1$. Furthermore, it is known that the initial critical exponent of a linearly recurrent infinite word is finite (see for instance [19]). It is also clear from the definition that such a word is written over a finite alphabet. We thus deduce from Theorem 2.1 the following result.

Theorem 2.2.4. Let $\mathbf{a}=\left(a_{\ell}\right)_{\ell \geq 0}$ be an aperiodic linearly recurrent sequence of positive integers. Then the real number $\xi:=\left[a_{0}, a_{1}, \ldots, a_{\ell}, \ldots\right]$ is a transcendental number satisfying the transcendence measure given in (2.1) and is thus either an $S$-number or a T-number.

\section{Transcendence measures for Maillet-Baker continued fractions}

In this section, we are interested in a class of quasi-periodic continued fractions introduced by Maillet [25] and studied by Baker [14, 15]. Baker proved in [14] the transcendence of these continued fractions by using an approach based on Roth's Theorem for number fields. His results were recently improved in [5] by means of a new application of the Subspace Theorem. Actually, the approach of [5] combined with the method initiated in [7] allows us to derive transcendence measures for such numbers.

We consider a real number $\xi:=\left[a_{0}, a_{1}, \ldots, a_{\ell}, \ldots\right]$, where $\mathbf{a}=\left(a_{\ell}\right)_{\ell \geq 0}$ is a noneventually periodic sequence of positive integers satisfying the following assumption: 
There exists an increasing sequence $\left(n_{k}\right)_{k \geq 0}$ of positive integers, and a sequence $\left(\lambda_{k}\right)_{k \geq 0}$ of positive integers, and a finite sequence $b_{1}, \ldots, b_{r}$ of positive integers, such that for every positive integer $k$, the sequence a begins with

$$
a_{0} a_{1} \ldots a_{n_{k}-1} \underbrace{B \ldots B}_{\lambda_{k} \text { times }}
$$

where $B:=b_{1} \ldots b_{r}$. It is understood here that

$$
a_{n_{k}-r} a_{n_{k}-r+1} \ldots a_{n_{k}-1} \neq B .
$$

We now state our main result regarding Maillet-Baker continued fractions.

Theorem 3.1. Let $\xi:=\left[a_{0}, a_{1}, \ldots, a_{\ell}, \ldots\right]$ be defined as above. Let $\left(p_{\ell} / q_{\ell}\right)_{\ell \geq 1}$ denote the sequence of convergents to $\xi$ and assume that the sequence $\left(q_{\ell}^{1 / \ell}\right)_{\ell \geq 1}$ is bounded. Assume further that

$$
\liminf _{k \rightarrow \infty} \lambda_{k} / n_{k}>0
$$

and

$$
\limsup _{k \rightarrow \infty} n_{k+1} / n_{k}<\infty
$$

Then $\xi$ is a transcendental number and there exists a constant $c$ independent of $d$ such that

$$
w_{d}(\xi) \leq \exp \left(c(\log 3 d)^{3}(\log \log 3 d)^{2}\right)
$$

for every positive integer $d$. In particular, $\xi$ is either an $S$-number or a $T$-number.

The proof of this result also relies on the quantitative version of the Subspace Theorem given in Section 6. As an illustration, we see that the real number

$$
\xi:=[1,2,2,1,2,1,1,1,2,1,1,1,1,1,1,1,2,1,1,1 \ldots],
$$

whose $n$-th partial quotient is equal to 2 if $n$ is a power of 2 and to 1 otherwise, is either an $S$-number or a $T$-number. Let us also remark that a Maillet-Baker continued fraction satisfying

$$
\limsup _{k \rightarrow \infty} \lambda_{k} / n_{k}=\infty
$$

corresponds to a $U_{2}$-number for it is extremely well approximated by the quadratic numbers

$$
\alpha_{k}:=\left[a_{0}, a_{1}, \ldots, a_{n_{k}-1}, B^{\infty}\right] .
$$

We note that such a situation cannot occur in Theorem 3.1 because of (3.2) and (3.4).

The proof of Theorem 3.1 can actually be slightly modified to obtain the following improvement of the Theorem of Baker mentioned in the introduction.

Theorem 3.2. Consider the quasi-periodic continued fraction

$$
\xi=[a_{0}, a_{1}, \ldots, a_{n_{0}-1}, \underbrace{a_{n_{0}}, \ldots, a_{n_{0}+r_{0}-1}}_{\lambda_{0} \text { times }}, \underbrace{a_{n_{1}}, \ldots, a_{n_{1}+r_{1}-1}}_{\lambda_{1} \text { times }}, \ldots],
$$


where the notation implies that $n_{k+1}=n_{k}+\lambda_{k} r_{k}$ and the $\lambda_{k}$ 's indicate the number of times a block of partial quotients is repeated. Suppose that the sequences $\left(a_{n}\right)_{n \geq 0}$ and $\left(r_{n}\right)_{n \geq 0}$ are bounded, that $\left(a_{n}\right)_{n \geq 0}$ is not ultimately periodic, and that

$$
\liminf _{k \rightarrow \infty} \lambda_{k+1} / \lambda_{k}>1
$$

If, moreover,

$$
\limsup _{k \rightarrow \infty} \lambda_{k+1} / \lambda_{k}<\infty
$$

then the real number $\xi$ is either an $S$-number or a $T$-number satisfying (3.5).

\section{Transcendence measures for palindromic continued fractions}

In this section, we are interested in continued fractions involving some symmetric patterns, whose study was initiated in [6].

We denote the mirror image of a finite word $W:=a_{1} \ldots a_{n}$ by $\bar{W}:=a_{n} \ldots a_{1}$. Then a finite word $W$ is a palindrome if $W=\bar{W}$. An infinite word that begins with arbitrarily large palindromes is termed palindromic. It was remarked in [6, Theorem 2.1] that real numbers whose continued fraction expansion is palindromic are either quadratic or transcendental.

Given a palindromic infinite word a, we denote by $\left(n_{k}\right)_{k \geq 1}$ the increasing sequence of all lengths of palindromic prefixes of $\mathbf{a}$. Then the palindromic density $d_{p}(\mathbf{a})$ of $\mathbf{a}$ is defined by

$$
d_{p}(\mathbf{a}):=\left(\limsup _{k \rightarrow \infty} n_{k+1} / n_{k}\right)^{-1} .
$$

This notion is for instance discussed in [23]. We refine Theorem 2.1 of [6] as follows.

Theorem 4.1. Let $\mathbf{a}=\left(a_{\ell}\right)_{\ell \geq 0}$ be a non-periodic sequence of positive integers with a positive palindromic density. Let $\left(p_{\ell} / q_{\ell}\right)_{\ell \geq 1}$ be the sequence of convergents to the real number $\xi:=\left[a_{0}, a_{1}, \ldots, a_{\ell}, \ldots\right]$, and assume that the sequence $\left(q_{\ell}^{1 / \ell}\right)_{\ell \geq 1}$ is bounded. If ice(a) is finite, then $\xi$ is either an $S$-number or a T-number; otherwise, $\xi$ is a $U_{2}$-number. Moreover, if ice(a) is finite, then there exists a constant $c$ independent of $d$ such that

$$
w_{d}(\xi) \leq \exp \left(c(\log 3 d)^{2}(\log \log 3 d)\right) \quad(d \geq 1) .
$$

Actually, it is not difficult to see that a sequence with a positive palindromic density satisfies Condition $(*)_{\widehat{w}}$ for some real number $w>1$; see for instance the proof of Theorem 4.6 of [3]. Consequently, we could deduce from Theorem 2.1 a result similar to Theorem 4.1 but with a weaker transcendence measure; namely, with (2.1) instead of (4.1). In particular, simultaneous approximations arising from palindromic patterns seem really needed to reach the transcendence measure given by (4.1). The proof of Theorem 4.1 is given in Section 9.

As an illustration of Theorem 4.1, we find that the Thue-Morse continued fraction $\xi_{\mathbf{t}}$ mentioned in the Introduction is either an $S$-number or a $T$-number, and it satisfies the transcendence measure given in (4.1). 


\section{Auxiliary results}

For the reader's convenience, we first recall some classical results from the theory of continued fractions, whose proofs can be found for example in the book of Perron [26].

Lemma 5.1. Let $\alpha=\left[a_{0}, a_{1}, \ldots, a_{n}, \ldots\right]$ and $\beta=\left[b_{0}, b_{1}, \ldots, b_{n}, \ldots\right]$ be two real numbers. Assume that there exists a positive integer $\ell$ such that $a_{i}=b_{i}$ for any $i=$ $0, \ldots, \ell$. Then $|\alpha-\beta| \leq q_{\ell}^{-2}$, where $q_{\ell}$ denotes the denominator of the $\ell$-th convergent to $\alpha$.

Lemma 5.2. Let $\alpha=\left[a_{0}, a_{1}, \ldots, a_{\ell}, \ldots\right]$ be a real number with convergents $\left(p_{\ell} / q_{\ell}\right)_{\ell \geq 1}$. Then, for any $\ell \geq 2$, we have

$$
q_{\ell-1} / q_{\ell}=\left[0, a_{\ell}, a_{\ell-1}, \ldots, a_{1}\right] .
$$

For positive integers $a_{1}, \ldots, a_{\ell}$, denote by $K_{\ell}\left(a_{1}, \ldots, a_{\ell}\right)$ the denominator of the rational number $\left[0, a_{1}, \ldots, a_{\ell}\right]$. It is commonly called a continuant.

Lemma 5.3. For any positive integers $a_{1}, \ldots, a_{\ell}$ and any integer $k$ with $1 \leq k \leq \ell-1$, we have

$$
K_{\ell}\left(a_{1}, \ldots, a_{\ell}\right)=K_{\ell}\left(a_{\ell}, \ldots, a_{1}\right)
$$

and

$$
\begin{aligned}
K_{k}\left(a_{1}, \ldots, a_{k}\right) \cdot K_{\ell-k}\left(a_{k+1}, \ldots, a_{\ell}\right) & \leq K_{\ell}\left(a_{1}, \ldots, a_{\ell}\right) \\
& \leq 2 K_{k}\left(a_{1}, \ldots, a_{k}\right) \cdot K_{\ell-k}\left(a_{k+1}, \ldots, a_{\ell}\right) .
\end{aligned}
$$

Lemma 5.4. Let $\left(a_{\ell}\right)_{\ell \geq 1}$ be a sequence of positive integers. For any positive integer $\ell$, we have

$$
K_{\ell}\left(a_{1}, \ldots, a_{\ell}\right) \geq 2^{(\ell-1) / 2} .
$$

We will also make use of the following four auxiliary results.

Lemma 5.5. Let $\alpha=\left[a_{0}, a_{1}, \ldots, a_{n}, \ldots\right]$ and $\beta=\left[b_{0}, b_{1}, \ldots, b_{n}, \ldots\right]$ be two real numbers whose convergents are respectively denoted by $\left(p_{n} / q_{n}\right)_{n \geq 1}$ and $\left(r_{n} / s_{n}\right)_{n \geq 1}$. Assume that both sequences $\left(q_{n}^{1 / n}\right)_{n \geq 1}$ and $\left(s_{n}^{1 / n}\right)_{n \geq 1}$ are bounded by a real number $M$. Assume that there exists a positive integer $\ell$ such that $a_{i}=b_{i}$ for any $i=0, \ldots, \ell$ and $a_{\ell+1} \neq b_{\ell+1}$. Then there exists a positive real number $\mu$, depending only on $M$, such that

$$
|\alpha-\beta| \geq 1 / q_{\ell}^{\mu}
$$

If we assume moreover that the partial quotients of $\beta$ are bounded by $M$, then there exists a positive real number $c$, depending only on $M$, such that

$$
|\alpha-\beta| \geq c / q_{\ell}^{2} \text {. }
$$

Proof. We follow the proof of Lemma 5 from [2]. The constants implicit in $\ll$ and $\gg$ below depend only on $M$. Set $\alpha^{\prime}=\left[a_{\ell+1}, a_{\ell+2}, \ldots\right]$ and $\beta^{\prime}=\left[b_{\ell+1}, b_{\ell+2}, \ldots\right]$. Since $a_{\ell+1} \neq b_{\ell+1}$, we have

$$
\left|\alpha^{\prime}-\beta^{\prime}\right| \geq \min \left\{\frac{1}{a_{\ell+2}+1}, \frac{1}{b_{\ell+2}+1}\right\} .
$$


Furthermore, our assumption on $\left(q_{n}^{1 / n}\right)_{n \geq 1}$ and $\left(s_{n}^{1 / n}\right)_{n \geq 1}$ implies that

$$
a_{\ell+1}, a_{\ell+2}, b_{\ell+1}, b_{\ell+2} \ll M^{\ell},
$$

hence

$$
\alpha^{\prime} \ll M^{\ell}, \quad \beta^{\prime} \ll M^{\ell} \text { and } \quad\left|\alpha^{\prime}-\beta^{\prime}\right| \gg M^{-\ell} .
$$

The theory of continued fractions gives that

$$
\alpha=\frac{p_{\ell} \alpha^{\prime}+p_{\ell-1}}{q_{\ell} \alpha^{\prime}+q_{\ell-1}} \quad \text { and } \quad \beta=\frac{p_{\ell} \beta^{\prime}+p_{\ell-1}}{q_{\ell} \beta^{\prime}+q_{\ell-1}},
$$

since the first $\ell$ partial quotients of $\alpha$ and $\beta$ are the same. We thus obtain

$$
|\alpha-\beta|=\left|\frac{p_{\ell} \alpha^{\prime}+p_{\ell-1}}{q_{\ell} \alpha^{\prime}+q_{\ell-1}}-\frac{p_{\ell} \beta^{\prime}+p_{\ell-1}}{q_{\ell} \beta^{\prime}+q_{\ell-1}}\right|=\left|\frac{\alpha^{\prime}-\beta^{\prime}}{\left(q_{\ell} \alpha^{\prime}+q_{\ell-1}\right)\left(q_{\ell} \beta^{\prime}+q_{\ell-1}\right)}\right| .
$$

Together with (5.1) and (5.2), this yields

$$
|\alpha-\beta| \gg M^{-3 \ell} q_{\ell}^{-2} \text {. }
$$

We infer from Lemma 5.4 that $q_{\ell} \geq 2^{(\ell-1) / 2}$. Combined with (5.4), this gives the first assertion of our lemma.

For the second assertion, we proceed as above, noticing that

$$
\left|\alpha^{\prime}-\beta^{\prime}\right| \geq 1-[0,1, M+1]=\frac{1}{M+2}
$$

and

$$
\left|\frac{\alpha^{\prime}-\beta^{\prime}}{q_{\ell} \alpha^{\prime}+q_{\ell-1}}\right| \geq \frac{1}{4(M+2)^{2} q_{\ell}} .
$$

The latter inequality follows from (5.5) if $\alpha^{\prime} \leq 2 \beta^{\prime}$, since then $\alpha^{\prime} \leq 2(M+1)$. If $\alpha^{\prime}>2 \beta^{\prime}$, then we simply observe that

$$
\left|\frac{\alpha^{\prime}-\beta^{\prime}}{q_{\ell} \alpha^{\prime}+q_{\ell-1}}\right| \geq \frac{\alpha^{\prime}}{2\left(q_{\ell} \alpha^{\prime}+q_{\ell-1}\right)} \geq \frac{1}{2\left(q_{\ell}+1\right)}
$$

to get (5.6). Since $\beta^{\prime} \leq M+1$, the second assertion of the lemma follows from (5.3) and (5.6).

Lemma 5.6. Let $\xi$ be a real number and $\left(p_{\ell} / q_{\ell}\right)_{\ell \geq 1}$ be the sequence of convergents to $\xi$. If the sequence $\left(q_{\ell}^{1 / \ell}\right)_{\ell \geq 1}$ is bounded, then $w_{1}(\xi)$ is finite, that is, $\xi$ is not a Liouville number.

Proof. Let $A$ be a positive real number such that $q_{\ell}<A^{\ell}$ for every positive integer $\ell$. By Lemma 5.4 we have $q_{\ell} \geq \sqrt{2}^{\ell-1}$ and thus there exists a positive real number $w>1$ such that $q_{\ell+1}<q_{\ell}^{w}$ for $\ell \geq 1$. 
By the theory of continued fractions,

$$
\left|\xi-\frac{p_{\ell}}{q_{\ell}}\right|>\frac{1}{2 q_{\ell} q_{\ell+1}}>\frac{1}{2 q_{\ell}^{w+1}}
$$

for every positive integer $\ell$. Since the best rational approximations to $\xi$ are in the sequence $\left(p_{\ell} / q_{\ell}\right)_{\ell \geq 1}$, we get $w_{1}(\xi) \leq w$. Consequently, $\xi$ is not a Liouville number.

We further state a classical Liouville inequality which can be found for instance in [17].

Lemma 5.7. Let $\beta$ be an algebraic number of degree $d$ and $P(X)$ a non-constant integer polynomial of degree $r$. Then, if $P(\beta) \neq 0$, we have

$$
|P(\beta)| \geq \frac{1}{(r+1)^{d-1}(d+1)^{r / 2} H(P)^{d-1} H(\beta)^{r}} .
$$

If $\alpha$ and $\beta$ are two non-zero distinct algebraic numbers of degree respectively $n$ and $m$, then

$$
|\alpha-\beta| \geq \frac{2 \max \left\{2^{-n}(n+1)^{-(m-1) / 2}, 2^{-m}(m+1)^{-(n-1) / 2}\right\}}{(n+1)^{m / 2}(m+1)^{n / 2} H(\alpha)^{m} H(\beta)^{n}} .
$$

Our last lemma gives a bound for the height of a quotient of algebraic numbers.

Lemma 5.8. Let $\alpha$ be a real algebraic number of degree $d \geq 2$. Let $x_{1}, x_{2}, x_{3}, x_{4}$ be integers with $\left(x_{3}, x_{4}\right) \neq(0,0)$ and of absolute values at most $A$. Then the height of the algebraic number $\beta=\left(x_{1} \alpha+x_{2}\right) /\left(x_{3} \alpha+x_{4}\right)$ is at most $2^{3(d+1)} H(\alpha)^{2} A^{2 d}$.

Proof. Using classical inequalities between the naïve height $H$, the Mahler measure $M$ and the logarithmic Weyl height h, we get

$$
\begin{aligned}
H(\beta) & \leq 2^{d} M(\beta) \leq 2^{d} e^{d \mathrm{~h}(\beta)} \leq 2^{d} e^{d \mathrm{~h}\left(x_{1} \alpha+x_{2}\right)} e^{d \mathrm{~h}\left(x_{3} \alpha+x_{4}\right)} \\
& \leq 2^{d} M\left(x_{1} \alpha+x_{2}\right) M\left(x_{3} \alpha+x_{4}\right) \leq 2^{d}(d+1) H\left(x_{1} \alpha+x_{2}\right) H\left(x_{3} \alpha+x_{4}\right) \\
& \leq 2^{3(d+1)} H(\alpha)^{2} A^{2 d}
\end{aligned}
$$

by Lemma A.4 from [17].

\section{The Quantitative Subspace Theorem}

The proofs of our result rely on the quantitative version of the Schmidt Subspace Theorem. The following statement was proved by Evertse [20]. We refer to [20] for the definition of the height of a linear form $L$, denoted by Height $(L)$. For our purpose, it is sufficient to note that it can be bounded from above in terms of the heights of the coefficients of $L$.

Theorem E. Let $m \geq 2, H$ and $d$ be positive integers. Let $L_{1}, \ldots, L_{m}$ be linearly independent (over $\overline{\mathbb{Q}}$ ) linear forms in $m$ variables with real algebraic coefficients. Assume that 
Height $\left(L_{i}\right) \leq H$ for $i=1, \ldots, m$ and that the number field generated by all the coefficients of these linear forms has degree at most $d$. Let $\varepsilon$ be a real number with $0<\varepsilon<1$. Then the primitive integer vectors $\mathbf{x}=\left(x_{1}, \ldots, x_{m}\right)$ in $\mathbb{Z}^{m}$ with $H(\mathbf{x}) \geq H$ and such that

$$
\prod_{i=1}^{m}\left|L_{i}(\mathbf{x})\right|<\left|\operatorname{det}\left(L_{1}, \ldots, L_{m}\right)\right| \cdot\left(\max \left\{\left|x_{1}\right|, \ldots,\left|x_{m}\right|\right\}\right)^{-\varepsilon}
$$

lie in at most

$$
c_{m, \varepsilon}(\log 4 d)(\log \log 4 d)
$$

proper subspaces of $\mathbb{Q}^{m}$, where $c_{m, \varepsilon}$ is a constant which only depends on $m$ and $\varepsilon$.

\section{Proof of Theorem 2.1}

We first show how the initial critical exponent allows us to control the approximation by quadratic numbers.

Lemma 7.1. Let $\mathbf{a}=\left(a_{\ell}\right)_{\ell \geq 1}$ be a non-periodic sequence of positive integers and set $\xi:=\left[0, a_{1}, \ldots, a_{\ell}, \ldots\right]$. Denote by $\left(p_{\ell} / q_{\ell}\right)_{\ell \geq 1}$ the sequence of convergents to $\xi$. Assume that the sequence $\left(q_{\ell}^{1 / \ell}\right)_{\ell \geq 1}$ is bounded and that ice $(\mathbf{a})=\infty$. Then $\xi$ is a $U_{2}$-number.

Proof. By assumption, the initial critical exponent of the sequence a is infinite. Consequently, for every positive integer $n$, there exists a finite word $V_{n}$ such that a begins with the word $V_{n}^{n}$. Set $\alpha_{n}=\left[0, V_{n}^{\infty}\right]$ and denote by $s_{n}$ the length of the word $V_{n}$. Since $\alpha_{n}$ is a root of the polynomial

$$
P_{n}(X):=q_{s_{n}-1} X^{2}+\left(q_{s_{n}}-p_{s_{n}-1}\right) X-p_{s_{n}},
$$

its height is at most equal to $q_{s_{n}}$. The first $n s_{n}$ partial quotients of $\xi$ and $\alpha_{n}$ being the same, we infer from Lemmas 5.1 and 5.3 that

$$
\left|\xi-\alpha_{n}\right|<q_{n s_{n}}^{-2} \leq q_{s_{n}}^{-2 n} \leq H\left(\alpha_{n}\right)^{-2 n} .
$$

Since $\mathbf{a}$ is a non-periodic sequence, the set $\left\{\alpha_{n}: n \geq 1\right\}$ is infinite, and (7.2) implies that $w_{2}(\xi)=\infty$. By Lemma 5.6, this shows that $\xi$ is a $U_{2}$-number.

The next lemma is essentially outlined at the end of [11].

Lemma 7.2. Let $\mathbf{a}=\left(a_{\ell}\right)_{\ell \geq 1}$ be a sequence of positive integers satisfying Condition $(*)_{\widehat{w}}$ for some $w>1$. Let $\left(p_{\ell} / q_{\ell}\right)_{\ell \geq 1}$ be the sequence of convergents to the real number $\xi:=\left[0, a_{1}, \ldots, a_{\ell}, \ldots\right]$. Assume that the sequence $\left(q_{\ell}^{1 / \ell}\right)_{\ell \geq 1}$ is bounded and that ice(a) is finite. Then $w_{2}(\xi)$ is finite.

Proof. Assume that the sequence of finite words $\left(V_{n}\right)_{n>1}$ and the real number $w$ arising from Condition $(*) \widehat{w}$ are fixed. We also assume that $\left|V_{1}\right| \geq 2$. Let $M \geq 3$ be a positive integer such that ice(a) $<M$ and $q_{\ell}<M^{\ell}$ for $\ell \geq 1$. Denote by $l_{n}$ the length of the word $V_{n}$ for $n \geq 1$. 
As in the proof of Lemma 7.1, we set $\alpha_{n}=\left[0, V_{n}^{\infty}\right]$ and note that $\alpha_{n}$ is a quadratic number whose height is at most $q_{l_{n}}$. Since the sequence $\left(q_{\ell}^{1 / \ell}\right)_{\ell \geq 1}$ is bounded and the first $\left\lfloor w l_{n}\right\rfloor$ partial quotients of $\xi$ and $\alpha_{n}$ are the same, we infer from Lemmas 5.1 and 5.3 that

$$
\left|\xi-\alpha_{n}\right|<q_{\left\lfloor w l_{n}\right\rfloor}^{-2}<q_{l_{n}}^{-2-\delta}
$$

for some positive real number $\delta$.

On the other hand, since ice $(\mathbf{a})<M$, the numbers $\alpha_{n}$ and $\xi$ cannot have the same first $M l_{n}$ partial quotients. The same observation applies for $\alpha_{n}$ and $\alpha_{n+1}$. Since the sequence $\left(q_{\ell}^{1 / \ell}\right)_{\ell \geq 1}$ is bounded by $M$, we infer from Lemmas 5.3 and 5.5 that there exists a positive real number $\lambda$, depending only on $M$, such that

$$
\left|\xi-\alpha_{n}\right|>q_{l_{n}}^{-\lambda}
$$

and

$$
\left|\alpha_{n+1}-\alpha_{n}\right|>q_{l_{n}}^{-\lambda}
$$

Let $N$ be a positive integer. Taking a subsequence of $\left(V_{n}\right)_{n \geq 1}$ if needed, we can assume that there exists a positive integer $C_{N}$, depending only on $N$ and on the maximum of the sequence $\left(\left|V_{m+1}\right| /\left|V_{m}\right|\right)_{m \geq 1}$, such that

$$
N\left|V_{n}\right|<\left|V_{n+1}\right|<C_{N}\left|V_{n}\right| \text { for } n \geq 1 .
$$

Then Lemma 5.4 implies that $q_{l_{n+1}}>2^{(N / 2)\left|V_{n}\right|}$, while, by assumption, $q_{l_{n}}<M^{\left|V_{n}\right|}$. Thus, if $N$ has been chosen large enough, we can guarantee that

$$
q_{l_{n+1}}>\max \left\{\left(2 q_{l_{n}}^{\lambda}\right)^{1 /(2+\delta)}, q_{l_{n}}^{2}\right\}
$$

for every positive integer $n$. A simple computation shows that it is sufficient to select

$$
N>\max \left\{\frac{4 \log M}{\log 2}, \frac{2 \lambda \log M}{(2+\delta) \log 2}+\frac{2}{2+\delta}\right\} .
$$

On the other hand, we infer from (7.6) that $q_{l_{n+1}}<M^{C_{N}\left|V_{n}\right|}$, while Lemma 5.4 implies that $q_{l_{n}}>2^{\left(\left|V_{n}\right|-1\right) / 2}$. This ensures the existence of a positive integer $C$, depending only on $C_{N}$ and $M$, such that

$$
q_{l_{n+1}}<q_{l_{n}}^{C}
$$

for every positive integer $n$. A simple computation shows that we can take $C=$ $6 C_{N} \log M$.

By (7.7) and (7.8), we will thus assume that

$$
\max \left\{\left(2 q_{l_{n}}^{\lambda}\right)^{1 /(2+\delta)}, q_{l_{n}}^{2}\right\}<q_{l_{n+1}}<q_{l_{n}}^{C}
$$

for every positive integer $n$.

Combining (7.3) for $\alpha_{n+1}$ with (7.5) and (7.9), we see that $2\left|\xi-\alpha_{n+1}\right|$ is at most $\left|\alpha_{n}-\alpha_{n+1}\right|$ and the triangle inequality then yields

$$
\left|\xi-\alpha_{n}\right| \geq \frac{1}{2}\left|\alpha_{n+1}-\alpha_{n}\right|
$$


Furthermore, if we set $c=1 /\left(4 \cdot 3^{5 / 2}\right)$, Lemma 5.7 and (7.10) give

$$
\left|\xi-\alpha_{n}\right| \geq c H\left(\alpha_{n}\right)^{-2} H\left(\alpha_{n+1}\right)^{-2} \geq c q_{l_{n}}^{-2} H\left(\alpha_{n+1}\right)^{-2} .
$$

We then infer from (7.3) and (7.9) that

$$
H\left(\alpha_{n+1}\right) \geq c^{1 / 2} q_{l_{n}}^{\delta / 2}>c^{1 / 2} q_{l_{n+1} /(2 C)} .
$$

For $n$ large enough, say $n \geq n_{0}$, we thus obtain

$$
q_{l_{n}}^{\delta /(3 C)}<H\left(\alpha_{n}\right) \leq q_{l_{n}} .
$$

Set $j:=\lceil\log (3 C / \delta) / \log 2\rceil$. Using inequality (7.9) leads to

$$
q_{l_{n}}^{\delta /(3 C)}<H\left(\alpha_{n}\right) \leq q_{l_{n}}<q_{l_{n+j} /(3 C)}^{\delta(}<H\left(\alpha_{n+j}\right) \leq q_{l_{n+j}}<q_{l_{n}}^{C^{j}}<H\left(\alpha_{n}\right)^{3 C^{j+1} / \delta} .
$$

We also deduce from (7.4) and (7.11) that

$$
\left|\xi-\alpha_{n}\right|>\frac{1}{H\left(\alpha_{n}\right)^{3 \lambda C / \delta}}
$$

for every integer $n \geq n_{0}$.

Set $\sigma=3 \lambda C / \delta, \theta=3 C^{j+1} / \delta$ and $\alpha_{n}^{\prime}=\alpha_{n_{0}+(n-1) j}$ for $n \geq 1$. We have shown the existence of a sequence $\left(\alpha_{n}^{\prime}\right)_{n \geq 1}$ of quadratic irrational numbers satisfying

(i) $H\left(\alpha_{n}^{\prime}\right)^{-\sigma}<\left|\xi-\alpha_{n}^{\prime}\right|<H\left(\alpha_{n}^{\prime}\right)^{-2-\delta}$;

(ii) $H\left(\alpha_{n}^{\prime}\right)<H\left(\alpha_{n+1}^{\prime}\right)<H\left(\alpha_{n}^{\prime}\right)^{\theta}$.

At this point, we are ready to prove that $w_{2}(\xi)$ is finite. Let us consider a quadratic irrational number $\alpha$ whose height satisfies

$$
H(\alpha)>\left(c H\left(\alpha_{1}^{\prime}\right)^{\delta}\right)^{1 / 2}
$$

If $\alpha \in\left\{\alpha_{n}^{\prime}: n \geq 1\right\}$, we simply use (i) to get

$$
|\xi-\alpha|>H(\alpha)^{-\sigma} \text {. }
$$

Now assume that $\alpha \notin\left\{\alpha_{n}^{\prime}: n \geq 1\right\}$. Then there exists a unique integer $n>1$ such that

$$
H\left(\alpha_{n-1}^{\prime}\right)<c^{1 / \delta} H(\alpha)^{2 / \delta}<H\left(\alpha_{n}^{\prime}\right) .
$$

In particular, inequality (ii) shows that

$$
H\left(\alpha_{n}^{\prime}\right)<c^{\theta / \delta} H(\alpha)^{2 \theta / \delta} .
$$

Since by assumption $\alpha$ and $\alpha_{n}^{\prime}$ denote two distinct quadratic numbers, Lemma 5.7 implies

$$
\left|\alpha-\alpha_{n}^{\prime}\right| \geq 2 c H(\alpha)^{-2} H\left(\alpha_{n}^{\prime}\right)^{-2} .
$$


We thus infer from (7.13) and (i) that $\left|\alpha-\alpha_{n}^{\prime}\right|>2\left|\xi-\alpha_{n}^{\prime}\right|$, hence,

$$
|\xi-\alpha| \geq|| \xi-\alpha_{n}^{\prime}|-| \alpha-\alpha_{n}^{\prime}|| \geq \frac{1}{2}\left|\alpha-\alpha_{n}^{\prime}\right| .
$$

We now deduce from (7.14) and (7.15) that

$$
|\xi-\alpha| \geq c^{1-2 \theta / \delta} H(\alpha)^{-2-4 \theta / \delta} .
$$

Together with (7.12), this shows that $w_{2}(\xi)$ is finite, concluding the proof.

We are now ready to prove Theorem 2.1.

Proof of Theorem 2.1. We keep the notation of Theorem 2.1. Let $\mathbf{a}=\left(a_{\ell}\right)_{\ell \geq 1}$ be a sequence of positive integers satisfying Condition $(*)_{\widehat{w}}$ for some $w>1$. Let $\left(p_{\ell} / q_{\ell}\right)_{\ell \geq 1}$ denote the sequence of convergents to the real number $\xi:=\left[0, a_{1}, \ldots, a_{\ell}, \ldots\right]$. Assume that the sequence $\left(q_{\ell}^{1 / \ell}\right)_{\ell \geq 1}$ is bounded by some constant $A$. By Lemma 5.6, $w_{1}(\xi)$ is finite. Thus, if ice(a) is infinite, then Lemma 7.1 implies that $\xi$ is a $U_{2}$-number, concluding the proof in that case.

From now on, we assume that ice $(\mathbf{a})$ is finite. We first observe that $w_{2}(\xi)$ is finite, in virtue of Lemma 7.2. Consequently, we thus only have to study the approximations to $\xi$ by algebraic numbers of degree at least three. Let $d \geq 3$ be an integer. Let $\alpha$ be an algebraic number of degree $d$. At several places in the proof below, it is convenient to assume that the height of $\alpha$ is sufficiently large. Let $\chi$ be a positive real number such that

$$
|\xi-\alpha|<H(\alpha)^{-\chi}
$$

Our aim is to find an upper bound for $\chi$ in terms of $d$. More precisely, we have to prove that

$$
\chi<\exp \left(c(\log 3 d)^{3}(\log \log 3 d)^{2}\right)
$$

for some constant $c$ which does not depend on $d$.

Taking if necessary a subsequence of $\left(V_{n}\right)_{n \geq 1}$, we can assume that there exists a constant $C$ such that

(iii') $\quad 2\left|V_{n}\right|<\left|V_{n+1}\right|<C\left|V_{n}\right|$ for every positive integer $n$.

Set $s_{n}=\left|V_{n}\right|$ for every positive integer $n$. Let $\kappa$ be the unique positive integer such that

$$
q_{s_{\kappa}} \leq H(\alpha)<q_{s_{\kappa+1}} .
$$

Denote by $M_{1}$ the largest integer such that $q_{s_{\kappa}}^{\chi}>q_{\left\lfloor w s_{\kappa}+M_{1}\right\rfloor}^{2}$ and observe that

$$
|\xi-\alpha|<q_{\left\lfloor w s_{\kappa+h}\right\rfloor}^{-2}
$$

for every $1 \leq h \leq M_{1}$. By definition of $M_{1}$, we have

$$
q_{s_{\kappa}}^{\chi}<q_{\left\lfloor w s_{\kappa+M}+1\right\rfloor}^{2} .
$$


Given a positive integer $\ell$, our assumption implies that $q_{\ell} \leq A^{\ell}$, while Lemma 5.4 ensures that $q_{\ell} \geq(\sqrt{2})^{\ell-1}$. We thus infer from (7.19) that

$$
(\sqrt{2})^{\chi\left(s_{\kappa}-1\right)} \leq A^{2 w s_{\kappa+M}+1}
$$

and, using (iii'), we obtain

$$
(\sqrt{2})^{\chi\left(s_{\kappa}-1\right)} \leq A^{2 w s_{\kappa} C^{M_{1}+1}} .
$$

Consequently, inequality (7.16) holds if we have

$$
M_{1}<c_{0}(\log 3 d)^{3}(\log \log 3 d)^{2}
$$

for some constant $c_{0}$ which does not depend on $d$.

We will argue by contradiction. From now on, we assume that

$$
M_{1}>c_{1}(\log 3 d)^{3}(\log \log 3 d)^{2}
$$

for some constant $c_{1}$, and we will derive a contradiction if $c_{1}$ is sufficiently large.

For every integer $n \geq 1$, set

$$
\alpha_{n}=\left[0, V_{n}^{\infty}\right]
$$

and observe as previously that $\alpha_{n}$ is a root of the quadratic polynomial $P_{n}(X)$ given by (7.1). Rolle's Theorem and Lemma 5.1 give that

$$
\left|P_{n}(\xi)\right|=\left|P_{n}(\xi)-P_{n}\left(\alpha_{n}\right)\right| \leq 3 q_{s_{n}}\left|\xi-\alpha_{n}\right|<3 q_{s_{n}} q_{\left\lfloor w s_{n}\right\rfloor}^{-2} \quad(n \geq 1)
$$

since condition (i) implies that the first $\left\lfloor w s_{n}\right\rfloor$ partial quotients of $\xi$ and $\alpha_{n}$ are the same. Furthermore, we infer from the theory of continued fractions that

$$
\left|q_{s_{n}} \xi-p_{s_{n}}\right|<q_{s_{n}}^{-1} \text { and }\left|q_{s_{n}-1} \xi-p_{s_{n}-1}\right|<q_{s_{n}}^{-1} .
$$

Using again Rolle's Theorem, inequalities (7.18) and (7.21) imply that

$$
\left|P_{\kappa+h}(\alpha)\right|<\left|P_{\kappa+h}(\xi)\right|+3 q_{s_{\kappa+h}}|\xi-\alpha|<6 q_{s_{\kappa+h}} q_{\left\lfloor w s_{\kappa+h}\right\rfloor}^{-2} \quad\left(1 \leq h \leq M_{1}\right) .
$$

Inequalities (7.18) and (7.22) ensure that, for $h=1, \ldots, M_{1}$,

$$
\left|q_{s_{\kappa+h}} \alpha-p_{s_{\kappa+h}}\right|<2 q_{s_{\kappa+h}}^{-1} \text { and }\left|q_{s_{\kappa+h}-1} \alpha-p_{s_{\kappa+h}-1}\right|<2 q_{s_{\kappa+h}}^{-1} .
$$

We are now going to apply Theorem $E$ to the following system of linear forms:

$$
\begin{aligned}
& L_{1}\left(X_{1}, X_{2}, X_{3}, X_{4}\right)=\alpha^{2} X_{2}+\alpha\left(X_{1}-X_{4}\right)-X_{3}, \\
& L_{2}\left(X_{1}, X_{2}, X_{3}, X_{4}\right)=\alpha X_{1}-X_{3}, \\
& L_{3}\left(X_{1}, X_{2}, X_{3}, X_{4}\right)=X_{1}, \\
& L_{4}\left(X_{1}, X_{2}, X_{3}, X_{4}\right)=X_{2} .
\end{aligned}
$$

Observe first that these linear forms are independent and with algebraic coefficients. Set $\mathcal{N}_{1}=\left\{s_{\kappa+h}:\left\lfloor M_{1} / 2\right\rfloor \leq h \leq M_{1}\right\}$ and $\mathcal{P}_{1}=\left\{\mathbf{p}_{n}=\left(q_{n}, q_{n-1}, p_{n}, p_{n-1}\right): n \in \mathcal{N}_{1}\right\}$. Let 
$n$ be in $\mathcal{N}_{1}$. Evaluating these linear forms at the integer point $\mathbf{p}_{n}$, we infer from (7.23) and (7.24) that

$$
\prod_{1 \leq i \leq 4}\left|L_{i}\left(\mathbf{p}_{n}\right)\right|<12 q_{n}^{2} q_{\lfloor w n\rfloor}^{-2} .
$$

Using again that $\sqrt{2}^{\ell-1} \leq q_{\ell} \leq A^{\ell}$ for every non-negative integer $\ell$, we get $q_{\lfloor w n\rfloor}>$ $q_{n}^{1+2 \eta}$ for some positive real number $\eta$, depending only on $w$ and on $A$. Thus, if the height of $\alpha$ is large enough, then $s_{\kappa}$ is itself sufficiently large to guarantee that

$$
\prod_{1 \leq i \leq 4}\left|L_{i}\left(\mathbf{p}_{n}\right)\right|<\left|\operatorname{det}\left(L_{1}, L_{2}, L_{3}, L_{4}\right)\right| q_{n}^{-\eta} .
$$

Furthermore, all elements of the set $\mathcal{P}_{1}$ are primitive (since $p_{n}$ and $q_{n}$ are always relatively prime) and we have $p_{n}>\operatorname{Height}\left(L_{i}\right)$ for $i=1, \ldots, 4$ and $n \in \mathcal{N}_{1}$. Moreover, the coefficients of the linear forms $L_{1}, \ldots, L_{4}$ generate a number field of degree $d$. We can thus apply Theorem E with $m=4$ and $\varepsilon=\eta$. Let $T_{1}$ be the upper bound given by (6.1) for the number of exceptional subspaces. Set

$$
M_{2}:=\left\lfloor M_{1} / T_{1}\right\rfloor .
$$

Since $\eta$ does not depend on the constant $c_{1}$, inequality (7.20) ensures the existence of a constant $c_{2}$ such that

$$
M_{2}>c_{2}(\log 3 d)^{2}(\log \log 3 d) .
$$

By the pigeonhole principle, there exists a proper subspace of $\mathbb{Q}^{4}$ containing at least $M_{2}$ points of $\mathcal{P}_{1}$. Thus, there exist a non-zero integer vector $\mathbf{x}=\left(x_{1}, x_{2}, x_{3}, x_{4}\right)$ and a set $\mathcal{N}_{2} \subset \mathcal{N}_{1}$ with cardinality $r \geq M_{2}$ such that

$$
x_{1} q_{n}+x_{2} q_{n-1}+x_{3} p_{n}+x_{4} p_{n-1}=0
$$

for every $n \in \mathcal{N}_{2}$. Let $l_{1}<\cdots<l_{r}$ denote the elements of $\mathcal{N}_{2}$.

We now make the following assumption that will be justified later.

Assumption $\mathcal{A}$. There exist three integers $1 \leq a<b<c \leq\lfloor r / 4\rfloor$ such that the vectors $\mathbf{p}_{l_{a}}, \mathbf{p}_{l_{b}}$ and $\mathbf{p}_{l_{c}}$ are linearly independent.

This makes possible the choice of $\mathbf{x}=\mathbf{p}_{l_{a}} \wedge \mathbf{p}_{l_{b}} \wedge \mathbf{p}_{l_{c}}$ in (7.26). Here, $\wedge$ denotes the vector product in $\mathbb{R}^{4}$. An easy computation shows that with this choice of $\mathbf{x}$ we have

$$
h_{1}:=\max \left\{\left|x_{1}\right|,\left|x_{2}\right|,\left|x_{3}\right|,\left|x_{4}\right|\right\} \leq 12 q_{l_{c}}^{3} .
$$

Let us remark now that $\left(x_{2}, x_{4}\right) \neq(0,0)$ since the vectors $\left(q_{n_{1}}, p_{n_{1}}\right)$ and $\left(q_{n_{2}}, p_{n_{2}}\right)$ are not collinear. Furthermore, $\alpha$ being irrational, the real number

$$
\beta:=-\frac{x_{1}+x_{3} \alpha}{x_{2}+x_{4} \alpha}
$$

is well-defined. It then follows from Lemma 5.8 and inequality (7.27) that

$$
H(\beta) \ll q_{l_{c}}^{6 d} H(\alpha)^{2},
$$


where, as below, the constant implied by $\ll$ depends only on $d$. Since $l_{c}>s_{\kappa+1}$, we infer from (7.17) that

$$
H(\alpha)<q_{l_{c}},
$$

and from (7.17) and (7.28) that

$$
H(\beta)<q_{l_{c}}^{9 d},
$$

if the height of $\alpha$ has been chosen large enough. By (7.26), we have

$$
\left|\beta-\frac{q_{n-1}}{q_{n}}\right|=\left|\frac{x_{1}+x_{3} \alpha}{x_{2}+x_{4} \alpha}-\frac{x_{1}+x_{3} p_{n} / q_{n}}{x_{2}+x_{4} p_{n-1} / q_{n-1}}\right|
$$

for every $n \in \mathcal{N}_{2}$. Using (7.22), we thus obtain

$$
\left|\beta-\frac{q_{n-1}}{q_{n}}\right| \ll \frac{h_{1}^{2}}{q_{n}^{2}\left|x_{2}+x_{4} \alpha\right|^{2}} .
$$

Then inequality (7.27) and Liouville's inequality given in Lemma 5.7 yield

$$
\left|\beta-\frac{q_{n-1}}{q_{n}}\right| \ll h_{1}^{2 d+2} H(\alpha)^{2} q_{n}^{-2} \ll q_{l_{c}}^{6(d+1)} H(\alpha)^{2} q_{n}^{-2} .
$$

Since $d \geq 3$, the height of $\alpha$ can be chosen large enough so that (7.29) implies

$$
\left|\beta-\frac{q_{n-1}}{q_{n}}\right|<q_{l_{c}}^{7(d+1)} q_{n}^{-2}
$$

for every $n \in \mathcal{N}_{2}$. Since by assumption $c \leq\lfloor r / 4\rfloor, l_{j+1}>2 l_{j}$ and $r \geq M_{2}$, we deduce from (7.25) that

$$
\left|q_{l_{j}} \beta-q_{l_{j}-1}\right|<q_{l_{j}}^{-1 / 2}
$$

for every $j$ with $\lfloor r / 2\rfloor \leq j \leq r$. Set $\mathcal{N}_{2}^{\prime}=\left\{n \in \mathcal{N}_{2}: n \geq l_{\lfloor r / 2\rfloor}\right\}$. Denote by $M_{2}^{\prime}$ the cardinality of this set and observe that $M_{2}^{\prime} \geq M_{2} / 2$.

We are now going to apply again Theorem E. Let us consider the following new system of linear forms:

$$
L_{1}^{\prime}\left(Y_{1}, Y_{2}, Y_{3}\right)=\beta Y_{1}-Y_{2}, \quad L_{2}^{\prime}\left(Y_{1}, Y_{2}, Y_{3}\right)=\alpha Y_{1}-Y_{3}, \quad L_{3}^{\prime}\left(Y_{1}, Y_{2}, Y_{3}\right)=Y_{1} .
$$

Observe that these linear forms have algebraic coefficients and are linearly independent. Furthermore, $\operatorname{det}\left(L_{1}^{\prime}, L_{2}^{\prime}, L_{3}^{\prime}\right)=1$ and the coefficients of these linear forms generate a field of degree $d$. Let us also remark that, by (7.30), we have $\operatorname{Height}\left(L_{i}^{\prime}\right)<q_{n}$ for $i=1,2,3$ and every $n \in \mathcal{N}_{2}^{\prime}$.

Let $n$ be in $\mathcal{N}_{2}^{\prime}$. Evaluating these linear forms at the primitive integer point $\left(q_{n}, q_{n-1}, p_{n}\right)$, we infer from (7.22) and (7.31) that

$$
\prod_{1 \leq i \leq 3}\left|L_{i}^{\prime}\left(q_{n}, q_{n-1}, p_{n}\right)\right|<q_{n}^{-1 / 2} .
$$


We can thus apply Theorem $\mathrm{E}$ with $\varepsilon=1 / 2$ and $m=3$. Let $T_{2}$ be the upper bound given in (6.1) for the number of exceptional subspaces. Set $M_{3}:=\left\lfloor T_{2} / M_{2}^{\prime}\right\rfloor$. Then inequality (7.25) implies the existence of a constant $c_{3}$ such that

$$
M_{3}>c_{3} \log d \text {. }
$$

By the pigeonhole principle, there exists a proper subspace of $\mathbb{Q}^{3}$ which contains at least $M_{3}$ points lying in the set $\mathcal{P}_{2}:=\left\{\mathbf{p}_{n}: n \in \mathcal{N}_{2}^{\prime}\right\}$. Thus, there exist a non-zero integer vector $\mathbf{y}=\left(y_{1}, y_{2}, y_{3}\right)$ and a set $\mathcal{N}_{3} \subset \mathcal{N}_{2}^{\prime}$ with cardinality $s \geq M_{3}$ such that

$$
y_{1} q_{n}+y_{2} q_{n-1}+y_{3} p_{n}=0
$$

for every $n \in \mathcal{N}_{3}$. Let $m_{1}<\cdots<m_{s}$ denote the elements of $\mathcal{N}_{3}$.

Observe that $\mathbf{p}_{1}^{\prime}=\left(q_{m_{1}}, q_{m_{1}-1}, p_{m_{1}}\right)$ and $\mathbf{p}_{2}^{\prime}=\left(q_{m_{2}}, q_{m_{2}-1}, p_{m_{2}}\right)$ are not collinear since $m_{2}>m_{1}>1$. This make possible the choice of $\mathbf{y}=\mathbf{p}_{1}^{\prime} \wedge \mathbf{p}_{2}^{\prime}$ in (7.33). Here and in the rest of the proof, $\wedge$ denotes the vector product in $\mathbb{R}^{3}$. Then an easy computation shows that

$$
h_{2}:=\max \left\{\left|y_{1}\right|,\left|y_{2}\right|,\left|y_{3}\right|\right\} \leq q_{m_{2}}^{2} .
$$

For every $n \in \mathcal{N}_{3}$, we can rewrite (7.33) as

$$
y_{1}+y_{2} \beta+y_{3} \alpha+y_{2}\left(\frac{q_{n-1}}{q_{n}}-\beta\right)+y_{3}\left(\frac{p_{n}}{q_{n}}-\alpha\right)=0 .
$$

In particular, for $n=m_{s}$, inequalities (7.22), (7.31) and (7.34) give

$$
\left|y_{1}+y_{2} \beta+y_{3} \alpha\right|<2 q_{m_{2}}^{2} q_{m_{s}}^{-3 / 2} .
$$

On the other hand, we have

$$
\left|y_{1}+y_{2} \beta+y_{3} \alpha\right|=\frac{\left|y_{3} x_{4} \alpha^{2}+\left(x_{2} y_{3}+x_{3} y_{2}+x_{4} y_{1}\right) \alpha+\left(x_{1} y_{2}+y_{1} x_{2}\right)\right|}{\left|x_{2}+x_{4} \alpha\right|} .
$$

Thus, if $y_{1}+y_{2} \beta+y_{3} \alpha \neq 0$, we infer from Liouville's inequality given in Lemma 5.7 that

$$
\left|y_{1}+y_{2} \beta+y_{3} \alpha\right| \gg h_{1}^{-d} h_{2}^{-d+1} H(\alpha)^{-2} .
$$

Using (7.17), (7.27), (7.34) and the fact that $m_{2}>l_{c}>s_{\kappa+1}$, the previous inequality gives

$$
\left|y_{1}+y_{2} \beta+y_{3} \alpha\right| \gg q_{m_{2}}^{-5 d} .
$$

By (7.32), we have $s \geq M_{3}>c_{3} \log d$ and since by assumption $m_{j}>2 m_{j-1}$, we can secure that $q_{m_{s}}>q_{m_{2}}^{7 d}$ and

$$
\left|y_{1}+y_{2} \beta+y_{3} \alpha\right|>q_{m_{2}}^{2} q_{m_{s}}^{-1},
$$

if $c_{3}$ is chosen large enough. Then (7.35) contradicts (7.36). Thereby, we get

$$
y_{1}+y_{2} \beta+y_{3} \alpha=0 .
$$


To reach the desired contradiction, we need another equation linking $\alpha$ and $\beta$. Actually, it is sufficient to slightly modify the previous system of linear forms and consider the system

$L_{1}^{\prime \prime}\left(Z_{1}, Z_{2}, Z_{3}\right)=\beta Z_{1}-Z_{2}, \quad L_{2}^{\prime \prime}\left(Z_{1}, Z_{2}, Z_{3}\right)=\alpha Z_{2}-Z_{3}, \quad L_{3}^{\prime \prime}\left(Z_{1}, Z_{2}, Z_{3}\right)=Z_{1}$.

Again, $\operatorname{det}\left(L_{1}^{\prime \prime}, L_{2}^{\prime \prime}, L_{3}^{\prime \prime}\right)=1$ and the coefficients of $L_{1}^{\prime \prime}, L_{2}^{\prime \prime}, L_{3}^{\prime \prime}$ generate a number field of degree $d$. Furthermore, Height $\left(L_{i}^{\prime \prime}\right)<q_{n}$ for $i=1,2,3$ and every $n \in \mathcal{N}_{2}^{\prime}$. Given an integer $n \in \mathcal{N}_{2}^{\prime}$ and evaluating these linear forms at the integer point $\left(q_{n}, q_{n-1}, p_{n-1}\right)$, we infer from (7.22) and (7.31) that

$$
\prod_{1 \leq i \leq 3}\left|L_{i}^{\prime \prime}\left(q_{n}, q_{n-1}, p_{n-1}\right)\right|<q_{n}^{-1 / 2} .
$$

We can apply Theorem E exactly as before and we will thus omit some details in what follows. The pigeonhole principle ensures the existence of a proper subspace of $\mathbb{Q}^{3}$ containing at least $M_{4}$ points of $\mathcal{P}_{2}^{\prime}$. Consequently, there exist a non-zero integer vector $\mathbf{z}=\left(z_{1}, z_{2}, z_{3}\right)$ and a set $\mathcal{N}_{4} \subset \mathcal{N}_{2}^{\prime}$ with cardinality $t \geq M_{4}$ such that

$$
z_{1} q_{n}+z_{2} q_{n-1}+z_{3} p_{n-1}=0,
$$

for every $n \in \mathcal{N}_{4}$. Let $n_{1}<\cdots<n_{t}$ denote the elements of $\mathcal{N}_{4}$.

Then inequality (7.34) can be replaced by

$$
\max \left\{\left|z_{1}\right|,\left|z_{2}\right|,\left|z_{3}\right|\right\} \leq q_{n_{2}}^{2},
$$

while (7.35) becomes

$$
\left|\frac{z_{1}}{\beta}+z_{2}+z_{3} \alpha\right|<2 q_{n_{2}}^{2} q_{n_{t}}^{-3 / 2} .
$$

Using Lemma 5.7, we argue as above to show that

$$
\frac{z_{1}}{\beta}+z_{2}+z_{3} \alpha=0
$$

if $c_{3}$ is sufficiently large.

Our last step is to show that equalities (7.37) and (7.38) provide a contradiction. Indeed, since $\alpha$ is irrational, $y_{2}$ is not zero and these equalities imply

$$
\left(z_{3} \alpha+z_{2}\right)\left(y_{3} \alpha+y_{1}\right)=y_{2} z_{1} .
$$

Now, if $y_{3} z_{3}=0$, then (7.37) and (7.38) imply that $\beta$ is rational. This gives a contradiction in virtue of (7.30) and (7.31). Consequently, we can assume that $y_{3} z_{3} \neq 0$. Then we find from (7.39) that $\alpha$ is a quadratic irrational. This contradicts the fact that $\alpha$ is an algebraic number of degree at least three. We have proved that the constant $c_{3}$ cannot be taken arbitrarily large. Thus, the constant $c_{1}$ in (7.20) is bounded as well. This ends the proof in the case where Assumption $\mathcal{A}$ is satisfied.

We turn now to the case where Assumption $\mathcal{A}$ is not satisfied. Since some steps are very close to the previous exposition, we just outline the proof. 
We return to the place where Assumption $\mathcal{A}$ has been introduced. Set $\mathcal{N}_{2}^{\prime \prime}=\left\{n \in \mathcal{N}_{2}\right.$ : $\left.l_{\lfloor r / 8\rfloor} \leq n \leq l_{\lfloor r / 4\rfloor}\right\}$ and $\mathcal{P}_{2}^{\prime \prime}=\left\{\mathbf{p}_{n}: n \in \mathcal{N}_{2}^{\prime \prime}\right\}$. Since Assumption $\mathcal{A}$ is not satisfied, all points lying in $\mathcal{P}_{2}^{\prime \prime}$ belong to the subspace generated by $\mathbf{p}_{l_{1}}$ and $\mathbf{p}_{l_{2}}$. Indeed, these vectors are clearly not collinear. In particular, if we set

$$
\left(\begin{array}{l}
y_{1}^{\prime} \\
y_{2}^{\prime} \\
y_{3}^{\prime}
\end{array}\right):=\left(\begin{array}{c}
q_{l_{1}} \\
q_{l_{1}-1} \\
p_{l_{1}}
\end{array}\right) \wedge\left(\begin{array}{c}
q_{l_{2}} \\
q l_{2}-1 \\
p_{l_{2}}
\end{array}\right),
$$

we see that $\left(y_{1}^{\prime}, y_{2}^{\prime}, y_{3}^{\prime}\right)$ is a non-zero integer vector such that

$$
y_{1}^{\prime} q_{n}+y_{2}^{\prime} q_{n-1}+y_{3}^{\prime} p_{n}=0
$$

for every integer $n \in \mathcal{N}_{2}^{\prime \prime}$. Moreover, an easy computation shows that

$$
h_{1}^{\prime}:=\max \left\{\left|y_{j}^{\prime}\right|: 1 \leq j \leq 3\right\}<q_{l_{2}}^{2} .
$$

Then we set

$$
\beta^{\prime}:=-\frac{y_{1}^{\prime}+y_{3}^{\prime} \alpha}{y_{2}^{\prime}}
$$

Note that $\beta^{\prime}$ is well-defined since it is easily checked that $y_{2}^{\prime}$ is not zero. Using now (7.40) and Lemma 5.8, we obtain

$$
H\left(\beta^{\prime}\right)<q_{l_{2}}^{3 d} .
$$

On the other hand, we can choose the height of $\alpha$ large enough to ensure that

$$
\left|q_{n} \beta^{\prime}-q_{n-1}\right|<q_{n}^{-1 / 2}
$$

for every $n \in \mathcal{N}_{2}^{\prime \prime}$. Since $\mathcal{N}_{2}^{\prime \prime}$ is a set with cardinality $M_{2}^{\prime \prime} \geq M_{2} / 8$, we infer from Lemma 5.7 and (7.41) that

$$
y_{1}^{\prime}+y_{2}^{\prime} \beta^{\prime}+y_{3}^{\prime} \alpha=0
$$

We set

$$
\left(\begin{array}{c}
z_{1}^{\prime} \\
z_{2}^{\prime} \\
z_{3}^{\prime}
\end{array}\right):=\left(\begin{array}{c}
q_{l_{1}} \\
q_{l_{1}-1} \\
p_{l_{1}-1}
\end{array}\right) \wedge\left(\begin{array}{c}
q_{l_{2}} \\
q_{l_{2}-1} \\
p_{l_{2}-1}
\end{array}\right) .
$$

The non-zero integer vector $\left(z_{1}^{\prime}, z_{2}^{\prime}, z_{3}^{\prime}\right)$ is such that

$$
z_{1}^{\prime} q_{n}+z_{2}^{\prime} q_{n-1}+z_{3}^{\prime} p_{n-1}=0
$$

for every $n \in \mathcal{N}_{2}^{\prime \prime}$. As previously, this leads to

$$
\frac{z_{1}^{\prime}}{\beta^{\prime}}+z_{2}^{\prime}+z_{3}^{\prime} \alpha=0 .
$$

We then obtain a contradiction using (7.42) and (7.43), exactly as in the case where Assumption $\mathcal{A}$ is satisfied. This concludes the proof of the main part of the theorem. 
We now briefly explain why the transcendence measure given by (2.1) can be improved to $(2.2)$ when a satisfies Condition $(*) \widehat{w}$ for some $w \geq 2$. In that case the quadratic numbers $\alpha_{n}$ introduced at the beginning of the present proof are very good approximations of $\xi$. More precisely, following the proof of Theorem 1 of [1], we obtain

$$
\left|\xi-\alpha_{n}\right| \ll H\left(\alpha_{n}\right)^{-4}
$$

On the other hand, we can assume that the $\alpha_{n}$ 's are all distinct and that

$$
\limsup _{n \rightarrow \infty} \frac{\log H\left(\alpha_{n+1}\right)}{\log H\left(\alpha_{n+1}\right)}<\infty .
$$

The desired transcendence measure then follows from Corollaire 4.3 of [7].

\section{Proofs of Theorems 3.1 and 3.2}

Proof of Theorem 3.1. We keep the notation of Theorem 3.1 and denote by $A \geq 3$ a strict upper bound for the sequence $\left(q_{\ell}^{1 / \ell}\right)_{\ell \geq 1}$. Without any loss of generality, we assume that $a_{0}=0$.

Let us consider the quadratic real number $\beta$ whose continued fraction expansion is purely periodic with period $\bar{B}=b_{r}, b_{r-1}, \ldots, b_{1}$, that is,

$$
\beta:=\left[b_{r}, b_{r-1}, \ldots, b_{1}, b_{r}, b_{r-1}, \ldots, b_{1}, \ldots\right]=[\bar{B}, \bar{B}, \ldots,] .
$$

Let $\left(r_{\ell} / s_{\ell}\right)_{\ell \geq 1}$ denote the sequence of convergents to $\beta$. For every positive integer $k$, set

$$
P_{k}:=p_{n_{k}+r \lambda_{k}}, Q_{k}:=q_{n_{k}+r \lambda_{k}}, P_{k}^{\prime}:=p_{n_{k}+r \lambda_{k}-1}, Q_{k}^{\prime}:=q_{n_{k}+r \lambda_{k}-1}, S_{k}:=s_{r \lambda_{k}} .
$$

As observed in Section 3, the assumptions (3.2) and (3.4) imply that

$$
\limsup _{k \rightarrow \infty} \lambda_{k} / n_{k}<\infty
$$

Consequently, taking a subsequence of $\left(n_{k}\right)_{k \geq 1}$ if necessary, our assumption (3.4) ensures the existence of a constant $C_{1}$ such that

$$
n_{k}+r \lambda_{k}<n_{k+1}<n_{k+1}+r \lambda_{k+1}<C_{1}\left(n_{k}+r \lambda_{k}\right)
$$

for every positive integer $k$. Let $N$ be a positive integer. Taking a subsequence of $\left(n_{k}\right)_{k \geq 1}$ if necessary, we can assume that there exists a constant $C_{N}$ depending on $N$ and $C_{1}$ such that

$$
N\left(n_{k}+r \lambda_{k}\right)<n_{k+1}+r \lambda_{k+1}<C_{N}\left(n_{k}+r \lambda_{k}\right)
$$

for every positive integer $k$. Then Lemma 5.4 implies that $Q_{k+1}>2^{\left(N\left(n_{k}+r \lambda_{k}\right)-1\right) / 2}$, while by assumption $Q_{k}<A^{n_{k}+r \lambda_{k}}$. Thus, if $N$ has been chosen large enough, we obtain

$$
Q_{k+1}>Q_{k}^{2}
$$


for every positive integer $k$. A simple computation shows that it is sufficient to choose $N>(2 \log A) / \log 2+1$.

On the other hand, we infer from (8.1) that $Q_{k+1}<A^{C_{N}\left(n_{k}+r \lambda_{k}\right)}$, while Lemma 5.4 implies that $Q_{k}>2^{\left(n_{k}+r \lambda_{k}-1\right) / 2}$. This ensures the existence of a positive integer $C$, depending only on $C_{N}$ and $A$, such that

$$
Q_{k+1}<Q_{k}^{C}
$$

for every positive integer $k$. A simple computation shows that we can take $C=6 C_{N} \log A$.

By (8.2) and (8.3), we will thus assume that

$$
Q_{k}^{2}<Q_{k+1}<Q_{k}^{C}
$$

for every positive integer $k$.

We also infer from (i) and (ii) that there exists a positive real number $v$ such that

$$
v<\lambda_{k} / n_{k}<1 / v
$$

for every positive integer $k$.

On the one hand, the theory of continued fractions gives

$$
\left|Q_{k} \xi-P_{k}\right|<1 / Q_{k} \text { and }\left|Q_{k}^{\prime} \xi-P_{k}^{\prime}\right|<1 / Q_{k}^{\prime},
$$

while, on the other hand, the assumption on a implies that

$$
P_{k} / Q_{k}=[0, a_{1}, \ldots, a_{n_{k}-1}, \underbrace{B, \ldots, B}_{\lambda_{k}}] .
$$

By Lemma 5.2, we obtain

$$
Q_{k}^{\prime} / Q_{k}=[0, \underbrace{\bar{B}, \ldots, \bar{B}}_{\lambda_{k}}, a_{n_{k}-1}, \ldots, a_{1}]
$$

and from Lemma 5.1 we get

$$
\left|Q_{k}^{\prime} \beta-Q_{k}\right|<Q_{k}^{\prime} / S_{k}^{2}
$$

Furthermore, we infer from (3.2) that $a_{n_{k}-1} \ldots a_{n_{k}-r} \neq \bar{B}$. Thus, Lemmas 5.3 and 5.5 imply that

$$
\left|\beta-\frac{Q_{k}}{Q_{k}^{\prime}}\right|>\frac{c_{\beta}}{S_{k}^{2}},
$$

where $c_{\beta}$ is a positive constant depending only on $\beta$.

Since the sequence $\left(q_{\ell}^{1 / \ell}\right)_{\ell \geq 1}$ is bounded, we infer from Lemma 5.6 that $w_{1}(\xi)$ is finite. We thus only have to deal with the approximations of $\xi$ by algebraic numbers of degree at least two. Let $d \geq 2$ be an integer. Let $\alpha$ be an algebraic number of degree $d$, the height of which will be chosen large enough. Let $\chi$ be a positive real number such that

$$
|\xi-\alpha|<H(\alpha)^{-\chi}
$$


Our aim is to find an upper bound for $\chi$ as a function of $d$. More precisely, we have to prove that

$$
\chi<\exp \left(c(\log 3 d)^{3}(\log \log 3 d)^{2}\right)
$$

for some constant $c$ which does not depend on $d$.

Let us denote by $k_{0}$ the unique positive integer such that

$$
Q_{k_{0}} \leq H(\alpha)<Q_{k_{0}+1}
$$

and by $M_{1}$ the largest integer such that $Q_{k_{0}}^{\chi}>Q_{k_{0}+M_{1}}^{2}$. Consequently,

$$
|\xi-\alpha|<Q_{k_{0}+h}^{-2}
$$

for every integer $h=1, \ldots, M_{1}$. If

$$
M_{1}<c_{0}(\log 3 d)^{3}(\log \log 3 d)^{2}
$$

for some constant $c_{0}$ which does not depend on $d$, then inequality (8.9) also holds, as shown by an easy computation using (8.4), (8.10) and (8.11).

We will argue by contradiction. From now on, we assume that

$$
M_{1}>c_{1}(\log 3 d)^{3}(\log \log 3 d)^{2}
$$

for some constant $c_{1}$, and we will derive a contradiction if $c_{1}$ is sufficiently large.

It follows from inequalities (8.6) and (8.11) that, for every integer $h=1, \ldots, M_{1}$,

$$
\left|Q_{k_{0}+h} \alpha-P_{k_{0}+h}\right|<2 / Q_{k_{0}+h} \quad \text { and } \quad\left|Q_{k_{0}+h}^{\prime} \alpha-P_{k_{0}+h}^{\prime}\right|<2 / Q_{k_{0}+h}^{\prime}
$$

We are now going to apply Theorem $\mathrm{E}$ to the following system of linear forms:

$$
\begin{aligned}
& L_{1}\left(X_{1}, X_{2}, X_{3}, X_{4}\right)=\alpha X_{1}-X_{3}, \\
& L_{2}\left(X_{1}, X_{2}, X_{3}, X_{4}\right)=\alpha X_{2}-X_{4}, \\
& L_{3}\left(X_{1}, X_{2}, X_{3}, X_{4}\right)=\beta X_{1}-X_{2}, \\
& L_{4}\left(X_{1}, X_{2}, X_{3}, X_{4}\right)=X_{1} .
\end{aligned}
$$

Observe that these linear forms are linearly independent over $\overline{\mathbb{Q}}$ and all have real algebraic coefficients. Set $\mathcal{N}_{1}=\left\{k_{0}+h:\left\lfloor M_{1} / 2\right\rfloor \leq h \leq M_{1}\right\}$ and $\mathcal{P}_{1}=\left\{\mathbf{p}_{k}=\left(Q_{k}, Q_{k}^{\prime}, P_{k}, P_{k}^{\prime}\right)\right.$ : $\left.k \in \mathcal{N}_{1}\right\}$. Let $k \in \mathcal{N}_{1}$. If we evaluate these linear forms at the integer point $\mathbf{p}_{k}$, inequalities (8.7) and (8.13) give

$$
\prod_{1 \leq i \leq 4}\left|L_{i}\left(\mathbf{p}_{k}\right)\right|<\frac{4}{S_{k}^{2}} .
$$

By assumption, $Q_{k} \leq A^{n_{k}+r \lambda_{k}}$, while Lemma 5.4 implies that $S_{k} \geq(\sqrt{2})^{r \lambda_{k}-1}$. We then infer from (8.5) that

$$
S_{k} \geq Q_{k}^{\eta}
$$


for some positive real number $\eta$, depending only on $r, v$ and $A$. If the height of $\alpha$ is large enough, we deduce from (8.14) and (8.15) that

$$
\prod_{1 \leq i \leq 4}\left|L_{i}\left(\mathbf{p}_{k}\right)\right|<\left|\operatorname{det}\left(L_{1}, L_{2}, L_{3}, L_{4}\right)\right| Q_{k}^{-\eta} .
$$

Since $P_{k}$ and $Q_{k}$ are relatively prime, every element in $\mathcal{P}_{1}$ is a primitive vector. Furthermore, the degree of the number field generated by the coefficients of the four linear forms is at most $2 d$ and $Q_{k}>\operatorname{Height}\left(L_{i}\right)$ for $i=1, \ldots, 4$ and $k \in \mathcal{N}_{1}$. We can thus apply Theorem E with $m=4$ and $\varepsilon=\eta$. Let $T_{1}$ be the upper bound given in (6.1) for the number of exceptional subspaces. Set

$$
M_{2}:=\left\lfloor M_{1} / T_{1}\right\rfloor
$$

Since $\eta$ does not depend on the choice of the constant $c_{1}$, inequality (8.12) ensures the existence of a constant $c_{2}$ which does not depend on $d$ and such that

$$
M_{2}>c_{2}(\log 3 d)^{2}(\log \log 3 d) .
$$

By the pigeonhole principle, there exists a proper subspace of $\mathbb{Q}^{4}$ containing at least $M_{2}$ points of $\mathcal{P}_{1}$. That is, there exist a non-zero integer vector $\mathbf{x}=\left(x_{1}, x_{2}, x_{3}, x_{4}\right)$ and a set $\mathcal{N}_{2} \subset \mathcal{N}_{1}$ with cardinality $s \geq M_{2}$ such that

$$
x_{1} Q_{k}+x_{2} Q_{k}^{\prime}+x_{3} P_{k}+x_{4} P_{k}^{\prime}=0
$$

for every $k \in \mathcal{N}_{2}$. Let $l_{1}<\cdots<l_{s}$ denote the elements of $\mathcal{N}_{2}$.

We now make the following assumption that will be justified later.

Assumption $\mathcal{A}$. There exist three integers $1 \leq a<b<c \leq\lfloor s / 4\rfloor$ such that the vectors $\mathbf{p}_{l_{a}}, \mathbf{p}_{l_{b}}$ and $\mathbf{p}_{l_{c}}$ are linearly independent.

This makes possible the choice of $\mathbf{x}=\mathbf{p}_{l_{a}} \wedge \mathbf{p}_{l_{b}} \wedge \mathbf{p}_{l_{c}}$ in equality (8.17). Here, $\wedge$ denotes the vector product in $\mathbb{R}^{4}$. As shown by an easy computation, this choice of $\mathbf{x}$ guarantees that

$$
h_{1}:=\max \left\{\left|x_{1}\right|,\left|x_{2}\right|,\left|x_{3}\right|,\left|x_{4}\right|\right\} \leq 12 Q_{l_{c}}^{3} .
$$

It thus follows from inequality (8.18) that

$$
\left|x_{4} \alpha Q_{k}^{\prime}+x_{1} Q_{k}+x_{2} Q_{k}^{\prime}+x_{3} P_{k}\right|=\left|x_{4}\left(Q_{k}^{\prime} \alpha-P_{k}^{\prime}\right)\right|<\left|x_{4}\right| / Q_{k}<12 Q_{l_{c}}^{3} / Q_{k}
$$

for every $k \in \mathcal{N}_{2}$.

Our next step consists in applying again Theorem $\mathrm{E}$ to the following new system of linear forms:

$$
\begin{aligned}
& L_{1}^{\prime}\left(X_{1}, X_{2}, X_{3}\right)=\alpha X_{1}-X_{3}, \\
& L_{2}^{\prime}\left(X_{1}, X_{2}, X_{3}\right)=x_{1} X_{1}+\left(x_{4} \alpha+x_{2}\right) X_{2}+x_{3} X_{3}, \\
& L_{3}^{\prime}\left(X_{1}, X_{2}, X_{3}\right)=X_{1} .
\end{aligned}
$$

We first observe that these linear forms have algebraic coefficients. We also deduce from (8.17) that $\left(x_{2}, x_{4}\right) \neq(0,0)$. Consequently, $x_{4} \alpha+x_{2} \neq 0$, since $\alpha$ is irrational. We 
then deduce that the linear forms are linearly independent. Furthermore, the number field generated by all the coefficients of these linear forms has degree $d$, and we infer from Lemma 5.7, (8.18) and (8.19) that

$$
\left|\operatorname{det}\left(L_{1}^{\prime}, L_{2}^{\prime}, L_{3}^{\prime}\right)\right|=\left|x_{4} \alpha+x_{2}\right|>Q_{l_{c}}^{-3 d}
$$

provided that $H(\alpha)$ is large enough.

Since by assumption $c \leq\lfloor s / 4\rfloor$ and $s \geq M_{2}$, we infer from (8.4) and (8.16) that the constant $c_{2}$ can be chosen large enough so that

$$
Q_{k}^{1 / 2}>12 Q_{l_{c}}^{3 d+3}
$$

for every $\lfloor s / 2\rfloor \leq k \leq s$. For the same reason, we can assume that $H\left(L_{i}^{\prime}\right)<Q_{k}$, for $i=1,2,3$ and every $\lfloor s / 2\rfloor \leq k \leq s$. Set $\mathcal{N}_{2}^{\prime}=\left\{n \in \mathcal{N}_{2}: n \geq l_{\lfloor s / 2\rfloor}\right\}$. Denote by $M_{2}^{\prime}$ the cardinality of this set and observe that

$$
M_{2}^{\prime} \geq M_{2} / 2
$$

Given an integer $k \in \mathcal{N}_{2}^{\prime}$, evaluating these linear forms at the primitive integer point $\mathbf{p}_{k}^{\prime}:=\left(Q_{k}, Q_{k}^{\prime}, P_{k}\right)$, we infer from (8.19), (8.20) and (8.21) that

$$
\prod_{1 \leq i \leq 3}\left|L_{i}^{\prime}\left(Q_{k}, Q_{k}^{\prime}, P_{k}\right)\right|<\left|\operatorname{det}\left(L_{1}^{\prime}, L_{2}^{\prime}, L_{3}^{\prime}\right)\right| Q_{k}^{-1 / 2}
$$

Furthermore, it follows from Lemma 5.7 that $P_{k}>\operatorname{Height}\left(L_{i}^{\prime}\right)$ for $i=1,2,3$. We can thus apply Theorem $\mathrm{E}$ with $\varepsilon=1 / 2$ and $m=3$. Let $T_{2}$ be the upper bound given in (6.1) for the number of exceptional subspaces. Set $M_{3}:=\left\lfloor T_{2} / M_{2}^{\prime}\right\rfloor$. Then (8.16) and (8.22) imply the existence of a constant $c_{3}$ such that

$$
M_{3}>c_{3} \log d .
$$

By the pigeonhole principle, there exists a proper subspace of $\mathbb{Q}^{3}$ which contains at least $M_{3}$ points lying in the set $\mathcal{P}_{2}:=\left\{\mathbf{p}_{k}^{\prime}=\left(Q_{k}, Q_{k}^{\prime}, P_{k}\right): k \in \mathcal{N}_{2}^{\prime}\right\}$. There thus exist a non-zero integer vector $\mathbf{y}=\left(y_{1}, y_{2}, y_{3}\right)$ and a set $\mathcal{N}_{3} \subset \mathcal{N}_{2}^{\prime}$ of cardinality $t \geq M_{3}$ such that

$$
y_{1} Q_{k}+y_{2} Q_{k}^{\prime}+y_{3} P_{k}=0
$$

for every $k \in \mathcal{N}_{3}$. Let $1 \leq m_{1}<\cdots<m_{t}$ denote the elements of $\mathcal{N}_{3}$.

Observe that $\mathbf{p}_{1}^{\prime}$ and $\mathbf{p}_{2}^{\prime}$ are not collinear. This make possible the choice of $\mathbf{y}=\mathbf{p}_{1}^{\prime} \wedge \mathbf{p}_{2}^{\prime}$ in (8.24). Here and in the rest of the proof, $\wedge$ denotes the vector product in $\mathbb{R}^{3}$. Then an easy computation shows that

$$
h_{2}:=\max \left\{\left|y_{1}\right|,\left|y_{2}\right|,\left|y_{3}\right|\right\} \leq Q_{m_{2}}^{2} .
$$

For every $k \in \mathcal{N}_{3}$, we can rewrite (8.24) as

$$
y_{1}+y_{2} \beta+y_{3} \alpha+y_{2}\left(\frac{Q_{k}^{\prime}}{Q_{k}}-\beta\right)+y_{3}\left(\frac{P_{k}}{Q_{k}}-\alpha\right)=0 .
$$


Choosing $k=m_{t}$, we can argue as in the proof of Theorem 2.1 and use (8.23) to deduce from the Liouville inequality given in Lemma 5.7 that

$$
y_{1}+y_{2} \beta+y_{3} \alpha=0 .
$$

As a particular instance of equality (8.26), we thus obtain

$$
y_{2}\left(\frac{Q_{m_{t}}^{\prime}}{Q_{m_{t}}}-\beta\right)=y_{3}\left(\frac{P_{m_{t}}}{Q_{m_{t}}}-\alpha\right) .
$$

It follows from Lemma 5.3 and (8.5) that there exists a real number $C_{1}>1$, depending only on $v$, such that

$$
Q_{m_{t}}>C_{1}^{m_{t}} S_{m_{t}}
$$

Consequently, on the one hand, we have

$$
\left|y_{3}\left(\frac{P_{m_{t}}}{Q_{m_{t}}}-\alpha\right)\right|<\frac{\left|y_{3}\right|}{Q_{m_{t}}^{2}}<\frac{\left|y_{3}\right|}{C_{1}^{2 m_{t}} S_{m_{t}}^{2}}<\frac{1}{C_{1}^{m_{t}}} \cdot \frac{1}{S_{m_{t}}^{2}},
$$

by (8.25), if $c_{3}$ is sufficiently large. On the other hand, we infer from (8.27) that $y_{2} \neq 0$ (since $\alpha$ is irrational), and (8.8) shows that

$$
\left|y_{2}\left(\frac{Q_{m_{t}}^{\prime}}{Q_{m_{t}}}-\beta\right)\right|>\frac{c_{\beta}}{S_{m_{t}}^{2}} .
$$

Inequalities (8.28) and (8.29) provide us with a contradiction as soon as $c_{3}$ is chosen large enough. This ends the proof in the case where Assumption $\mathcal{A}$ is satisfied.

We now outline the opposite case. We return to the place where Assumption $\mathcal{A}$ has been introduced. Set $\mathcal{N}_{2}^{\prime \prime}=\left\{n \in \mathcal{N}_{2}: l_{\lfloor r / 8\rfloor} \leq n \leq l_{\lfloor r / 4\rfloor}\right\}$ and $\mathcal{P}_{2}^{\prime \prime}=\left\{\mathbf{p}_{n}: n \in \mathcal{N}_{2}^{\prime \prime}\right\}$. Since Assumption $\mathcal{A}$ is not satisfied, all points lying in $\mathcal{P}_{2}^{\prime \prime}$ belong to the vector plane generated by $\mathbf{p}_{l_{1}}$ and $\mathbf{p}_{l_{2}}$. Indeed, these vectors are clearly not collinear. In particular, if we set

$$
\left(\begin{array}{c}
y_{1}^{\prime} \\
y_{2}^{\prime} \\
y_{3}^{\prime}
\end{array}\right):=\left(\begin{array}{c}
q_{l_{1}} \\
q_{l_{1}-1} \\
p_{l_{1}}
\end{array}\right) \wedge\left(\begin{array}{c}
q_{l_{2}} \\
q_{l_{2}-1} \\
p_{l_{2}}
\end{array}\right)
$$

we find that $\left(y_{1}^{\prime}, y_{2}^{\prime}, y_{3}^{\prime}\right)$ is a non-zero integer vector such that

$$
y_{1}^{\prime} Q_{k}+y_{2}^{\prime} Q_{k}^{\prime}+y_{3}^{\prime} P_{k}=0
$$

for every integer $k \in \mathcal{N}_{2}^{\prime \prime}$. Moreover, an easy computation shows that

$$
h_{1}^{\prime}:=\max \left\{\left|y_{j}^{\prime}\right|: 1 \leq j \leq 3\right\}<Q_{l_{2}}^{2} .
$$

We are now in the same situation as in (8.25). We can thus argue exactly as previously to conclude the proof of the theorem.

Proof of Theorem 3.2. The proof follows essentially the same lines as that of Theorem 3.1 , with, however, some modification that we point out below. 
Since the sequences $\left(a_{n}\right)_{n \geq 0}$ and $\left(r_{n}\right)_{n \geq 0}$ are bounded, there exists a finite set $\mathcal{B}=$ $\left\{B_{1}, \ldots, B_{m}\right\}$ of finite words such that all the finite words $a_{n_{k}} a_{n_{k}+1} \ldots a_{n_{k}+r_{k}-1}$ belong to $\mathcal{B}$.

For $j=1, \ldots, m$, set

$$
\beta_{j}:=\left[\overline{B_{j}}, \overline{B_{j}}, \ldots\right]
$$

Let $\left(r_{\ell}^{(j)} / s_{\ell}^{(j)}\right)_{\ell \geq 1}$ denote the sequence of convergents to $\beta_{j}$. For every positive integer $k$, set

$$
P_{k}:=p_{n_{k}+r_{k} \lambda_{k}}, Q_{k}:=q_{n_{k}+r_{k} \lambda_{k}}, P_{k}^{\prime}:=p_{n_{k}+r_{k} \lambda_{k}-1}, Q_{k}^{\prime}:=q_{n_{k}+r_{k} \lambda_{k}-1}, S_{k}:=s_{r_{k} \lambda_{k}}^{(j)},
$$

where the index $j$ is such that $a_{n_{k}} a_{n_{k}+1} \ldots a_{n_{k}+r_{k}-1}=B_{j}$.

Then we follow the proof of Theorem 3.1 and consider, for $j=1, \ldots, m$, the following system of linear forms:

$$
\begin{aligned}
& L_{1}^{(j)}\left(X_{1}, X_{2}, X_{3}, X_{4}\right)=\alpha X_{1}-X_{3}, \\
& L_{2}^{(j)}\left(X_{1}, X_{2}, X_{3}, X_{4}\right)=\alpha X_{2}-X_{4}, \\
& L_{3}^{(j)}\left(X_{1}, X_{2}, X_{3}, X_{4}\right)=\beta_{j} X_{1}-X_{2}, \\
& L_{4}^{(j)}\left(X_{1}, X_{2}, X_{3}, X_{4}\right)=X_{1} .
\end{aligned}
$$

Similar arguments to those above show that, for $k$ in $\mathcal{N}_{1}$, there exist an absolute positive number $\eta$ and an index $j$ in $\{1, \ldots, m\}$ such that

$$
\prod_{1 \leq i \leq 4}\left|L_{i}^{(j)}\left(\mathbf{p}_{k}\right)\right|<\left|\operatorname{det}\left(L_{1}^{(j)}, L_{2}^{(j)}, L_{3}^{(j)}, L_{4}^{(j)}\right)\right| Q_{k}^{-\eta} .
$$

By the pigeonhole principle, there exist a non-zero integer vector $\mathbf{z}=\left(z_{1}, z_{2}, z_{3}, z_{4}\right)$, an index $j$ in $\{1, \ldots, m\}$, and a set $\mathcal{N}_{3} \subset \mathcal{N}_{1}$ of cardinality at least $\left\lfloor M_{2} / m\right\rfloor$ (with $M_{2}$ defined in (8.16)) such that

$$
z_{1} Q_{k}+z_{2} Q_{k}^{\prime}+z_{3} P_{k}+z_{4} P_{k}^{\prime}=0
$$

and $a_{n_{k}} a_{n_{k}+1} \ldots a_{n_{k}+r_{k}-1}=B_{j}$ for every $k \in \mathcal{N}_{3}$.

From now on, we simply follow the proof of Theorem 3.1, with $\beta$ replaced by $\beta_{j}$ in (8.26) and below. This proves Theorem 3.2.

\section{Proof of Theorem 4.1}

In this section, we explain how Theorem 4.1 can be deduced from Theorem 2.1 and Théorème 4.1 of [7].

Proof of Theorem 4.1. Let $\mathbf{a}=\left(a_{\ell}\right)_{\ell \geq 1}$ be a sequence of positive integers satisfying the assumptions of Theorem 4.1. In particular, a has a positive palindromic density and we denote by $\left(n_{k}\right)_{k \geq 1}$ the increasing sequence of all lengths of palindromic prefixes of $\mathbf{a}$. Set $\xi=\left[0, a_{1}, \ldots, a_{\ell}, \ldots\right]$ and denote as usual by $p_{\ell} / q_{\ell}$ the $\ell$-th convergent to $\xi$. 
As already mentioned in Section 4 , the fact that a satisfies Condition $(*)_{\widehat{w}}$ for some $w>1$ can be established by following the proof of Theorem 4.6 of [3]. It thus follows from Theorem 2.1 that $\xi$ is a $U_{2}$-number if ice(a) is infinite, while it is either an $S$-number or a $T$-number otherwise.

From now on, we assume that ice(a) is finite. Since the word $a_{1} \ldots a_{n_{k}}$ is a palindrome, Lemma 5.2 implies that $p_{n_{k}}=q_{n_{k}-1}$. Recalling that

$$
\left|\xi-\frac{p_{n_{k}}}{q_{n_{k}}}\right|<\frac{1}{q_{n_{k}}^{2}} \quad \text { and } \quad\left|\xi-\frac{p_{n_{k}-1}}{q_{n_{k}-1}}\right|<\frac{1}{q_{n_{k}-1}^{2}}
$$

we infer from $p_{n_{k}}=q_{n_{k}-1}, 0<\xi<1, a_{1}=a_{n_{k}},\left|p_{n_{k}} q_{n_{k}-1}-p_{n_{k}-1} q_{n_{k}}\right|=1$ and $q_{n_{k}} \leq\left(a_{n_{k}}+1\right) q_{n_{k}-1}$ that

$$
\begin{aligned}
\left|\xi^{2}-\frac{p_{n_{k}-1}}{q_{n_{k}}}\right| & =\left|\xi^{2}-\frac{p_{n_{k}-1}}{q_{n_{k}-1}} \cdot \frac{p_{n_{k}}}{q_{n_{k}}}\right| \leq\left|\xi+\frac{p_{n_{k}-1}}{q_{n_{k}-1}}\right| \cdot\left|\xi-\frac{p_{n_{k}}}{q_{n_{k}}}\right|+\frac{1}{q_{n_{k}} q_{n_{k}-1}} \\
& \leq 2\left|\xi-\frac{p_{n_{k}}}{q_{n_{k}}}\right|+\frac{1}{q_{n_{k}} q_{n_{k}-1}}<\frac{a_{1}+3}{q_{n_{k}}^{2}} .
\end{aligned}
$$

Consequently, there exists an increasing sequence $\left(q_{n_{k}}\right)_{k \geq 1}$ of positive integers such that

$$
\left\|q_{n_{k}} \xi\right\| \cdot\left\|q_{n_{k}} \xi^{2}\right\| \ll 1 / q_{n_{k}}^{2}
$$

where $\|\cdot\|$ denotes the distance to the nearest integer. Furthermore, since $\mathbf{a}$ has a positive palindromic density and the sequence $\left(q_{\ell}^{1 / \ell}\right)_{\ell \geq 1}$ is bounded, Lemma 5.4 ensures that

$$
\limsup _{k \rightarrow \infty} \frac{\log q_{n_{k+1}}}{\log q_{n_{k}}}<\infty .
$$

The transcendence measure given in (4.1) is then obtained as a consequence of Théorème 4.1 from [7]. This concludes the proof.

\section{Proofs of Theorems 2.2.1 and 2.2.2}

In this section, we explain how Theorems 2.2.1 and 2.2.2 can be deduced from Theorem 2.1 .

Proof of Theorem 2.2.1. Let $\mathbf{a}=\left(a_{\ell}\right)_{\ell \geq 0}$ be a Sturmian sequence of slope $\alpha$ and let $a$ and $b$ be the two integer values taken by $\mathbf{a}$.

If $\alpha$ has bounded partial quotients, it is proved in [19] that $\mathbf{a}$ is linearly recurrent. We can thus apply Theorem 2.2.4 to conclude the proof in that case.

If $\alpha$ has unbounded partial quotients, the key auxiliary ingredient is Proposition 11.1 of [8]. It asserts that, for every integer $m$, there exist finite words $U_{m}, V_{m}$ and a positive real number $s_{m}$ such that $U_{m} V_{m}^{s_{m}}$ is a prefix of a and

$$
\left|U_{m} V_{m}^{s_{m}}\right| /\left|U_{m} V_{m}\right| \geq m .
$$


In this case, the quadratic real number $\xi_{m}$ whose continued fraction expansion is given be the ultimately periodic infinite word $U_{m} V_{m}^{\infty}$ is a very good approximation to $\xi$. More precisely, since the height of $\xi_{m}$ is at most $(a+b)^{\left|U_{m} V_{m}\right|}$ and, by Lemmas 5.1 and 5.4,

$$
\left|\xi-\xi_{m}\right| \leq 2^{-\left|U_{m}+s_{m} V_{m}\right|+1}
$$

we deduce from (10.1) that there exists a positive real number $C$ independent of $m$ and such that

$$
\left|\xi-\xi_{m}\right| \leq H\left(\xi_{m}\right)^{-C m}
$$

Consequently, if $\alpha$ has unbounded partial quotients, then $\xi$ is a $U_{2}$-number.

Proof of Theorem 2.2.2. Let a be a non-trivial recurrent fixed point of a morphism $\sigma$ defined over a finite set $\mathcal{A}$. In view of Theorem 2.1 , it is sufficient to prove that

- the sequence a satisfies Condition $(*)_{\widehat{w}}$ for some real number $w>1$;

- ice(a) is finite.

For the first point, we refer the reader to the proof of Theorem 3 of [1]. It thus remains to prove that ice(a) is finite. Define the length of the morphism $\sigma$ by

$$
|\sigma|:=\max \{|\sigma(a)|: a \in \mathcal{A}\}
$$

We will actually prove that ice $(\mathbf{a}) \leq 2|\sigma|$.

Assume that this inequality does not hold and set $N=2|\sigma|$. There exists a non-empty word $U$ such that $U^{N}$ is a prefix of a. Without loss of generality we can assume that $U$ has been chosen with minimal length. Since a is fixed by $\sigma$, the word $\sigma(U)$ is a prefix of $\mathbf{a}$. We observe that $N$ is large enough to ensure that $\sigma(U)$ is also a prefix of $U^{N}$. There thus exist a non-negative integer $r$ and a (possibly empty) prefix $U_{1}$ of $U$ such that $\left|U_{1}\right|<|U|$ and

$$
\sigma(U)=U^{r} U_{1}
$$

If $U_{1}$ is the empty word, then we deduce that $\mathbf{a}=U^{\infty}$, providing a contradiction with the fact that $\mathbf{a}$ is a non-eventually periodic sequence.

From now on, we assume that $U_{1}$ is not the empty word. Since $U^{2}$ is a prefix of a, the word $\sigma\left(U^{2}\right)$ is also a prefix of $\mathbf{a}$, and we observe that $N$ is large enough to guarantee that $\sigma\left(U^{2}\right)$ is a prefix of $U^{N}$. From (10.2), we infer that $U^{r} U_{1} U^{r} U_{1}$ is a prefix of $U^{N}$. Let $U_{2}$ denote the prefix of $U$ of length $|U|-\left|U_{1}\right|$ and $U_{3}$ denote the suffix of $U$ of length $\left|U_{1}\right|$, so that $U=U_{2} U_{3}$. Since $U^{r} U_{1} U^{r} U_{1}$ is a prefix of $U^{N}$, we deduce that $U=U_{1} U_{2}=U_{3} U_{2}$. Consequently, $U_{2}$ and $U_{3}$ commute. Since $U_{1}$ and $U_{2}$ are nonempty, there exist a word $V$ and an integer $m \geq 2$ such that $U=V^{m}$. In particular, the sequence a begins with the word $V^{N}$. This contradicts the fact that $U$ has been chosen with minimal length, concluding the proof.

Acknowledgments. We are very much indebted to the referee, who pointed out several obscure points in a first version of the present text. 


\section{References}

[1] Adamczewski, B., Bugeaud, Y.: On the complexity of algebraic numbers II. Continued fractions. Acta Math. 195, 1-20 (2005) Zbl pre05039002 MR 2233683

[2] Adamczewski, B., Bugeaud, Y.: On the Littlewood conjecture in simultaneous Diophantine approximation. J. London Math. Soc. 73, 355-366 (2006) Zbl 1093.11052 MR 2225491

[3] Adamczewski, B., Bugeaud, Y.: Real and $p$-adic expansions involving symmetric patterns. Int. Math. Res. Notices 2006, art. ID 75968, 17 pp. Zbl 1113.11041 MR 2250005

[4] Adamczewski, B., Bugeaud, Y.: On the complexity of algebraic numbers I. Expansions in integer bases. Ann. of Math. 165, 547-565 (2007) Zbl pre05180742 MR 2299740

[5] Adamczewski, B., Bugeaud, Y.: On the Maillet-Baker continued fractions. J. Reine Angew. Math. 606, 105-121 (2007) Zbl 1145.11054 MR 2337643

[6] Adamczewski, B., Bugeaud, Y.: Palindromic continued fractions. Ann. Inst. Fourier (Grenoble) 57, 1557-1574 (2007) Zbl 1126.11036 MR 2364142

[7] Adamczewski, B., Bugeaud, Y.: Mesures de transcendance et aspects quantitatifs de la méthode de Thue-Siegel-Roth-Schmidt. Proc. London Math. Soc. (2010), doi: 10.1112/plms/pdp054

[8] Adamczewski, B., Bugeaud, Y.: Nombres réels de complexité sous-linéaire : mesures d'irrationalité et de transcendance. J. Reine Angew. Math., to appear

[9] Adamczewski, B., Bugeaud, Y., Davison, J. L.: Continued fractions and transcendental numbers. Ann. Inst. Fourier (Grenoble) 56, 2093-2113 (2006) Zbl 1152.11034 MR 2290775

[10] Adamczewski, B., Bugeaud, Y., Luca, F.: Sur la complexité des nombres algébriques. C. R. Math. Acad. Sci. Paris 339, 11-14 (2004) Zbl 1119.11019 MR 2075225

[11] Adamczewski, B., Cassaigne, J.: Diophantine properties of real numbers generated by finite automata. Compos. Math. 142, 1351-1372 (2006) Zbl 1134.11011 MR 2278750

[12] Allouche, J.-P., Davison, J. L., Queffélec, M., Zamboni, L. Q.: Transcendence of Sturmian or morphic continued fractions. J. Number Theory 91, 39-66 (2001) Zbl 0998.11036 MR 1869317

[13] Allouche, J.-P., Shallit, J.: Automatic Sequences: Theory, Applications, Generalizations. Cambridge Univ. Press, Cambridge (2003) Zbl 1086.11015 MR 1997038

[14] Baker, A.: Continued fractions of transcendental numbers. Mathematika 9, 1-8 (1962) Zbl 0105.03903 MR 0144853

[15] Baker, A.: On Mahler's classification of transcendental numbers. Acta Math. 111, 97-120 (1964) Zbl 0147.03403 MR 0157943

[16] Berthé, V., Holton, C., Zamboni, L. Q.: Initial powers of Sturmian words. Acta Arith. 122, 315-347 (2006) Zbl 1117.37005 MR 2234421

[17] Bugeaud, Y.: Approximation by Algebraic Numbers. Cambridge Tracts in Math. 160, Cambridge (2004) Zbl 1055.11002 MR 2136100

[18] Davison, J. L.: Quasi-periodic continued fractions. J. Number Theory 127, 272-282 (2007) Zbl pre05231964 MR 2362436

[19] Durand, F.: Linearly recurrent subshifts have a finite number of non-periodic subshift factors. Ergodic Theory Dynam. Systems 20, 1061-1078 (2000) Zbl 0965.37013 MR 1779393

[20] Evertse, J.-H.: An improvement of the quantitative subspace theorem. Compos. Math. 101, 225-311 (1996) Zbl 0856.11030 MR 1394517

[21] Feldman, N. I., Nesterenko, Yu. V.: Number Theory. IV. Transcendental Numbers. Encyclopaedia Math. Sci. 44, Springer, Berlin (1998) Zbl 0885.11004 MR 1603604

[22] Ferenczi, S., Mauduit, Ch.: Transcendence of numbers with a low complexity expansion.

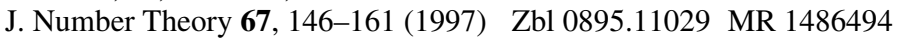


[23] Fischler, S.: Palindromic prefixes and episturmian words. J. Combin. Theory Ser. A 113, 1281-1304 (2006) Zbl 1109.68082 MR 2259061

[24] Mahler, K.: Zur Approximation der Exponentialfunktionen und des Logarithmus. I, II. J. Reine Angew. Math. 166, 118-150 (1932) JFM 57.0242.03 (I), JFM 58.0207.01 (II)

[25] Maillet, E.: Introduction à la théorie des nombres transcendants et des propriétés arithmétiques des fonctions. Gauthier-Villars, Paris (1906) Zbl 37.0237.02

[26] Perron, O.: Die Lehre von den Kettenbrüchen. Teubner, Leipzig (1929) JFM 55.0262.09

[27] Queffélec, M.: Transcendance des fractions continues de Thue-Morse. J. Number Theory 73, 201-211 (1998) Zbl 0920.11045 MR 1658023

[28] Ridout, D.: Rational approximations to algebraic numbers. Mathematika 4, 125-131 (1957) Zbl 0079.27401 MR 0093508

[29] Roth, K. F.: Rational approximations to algebraic numbers. Mathematika 2, 1-20 (1955); corrigendum, ibid., 168 Zbl 0064.28501 MR 0072182

[30] Schmidt, W. M.: Norm form equations. Ann. of Math. 96, 526-551 (1972) Zbl 0226.10024 MR 0314761

[31] Schmidt, W. M.: Diophantine Approximation. Lecture Notes in Math. 785, Springer (1980) Zbl 0421.10019 MR 0568710

[32] Schmidt, W. M.: The subspace theorem in Diophantine approximation. Compos. Math. 69, 121-173 (1989) Zbl 0683.10027 MR 0984633

[33] Schneider, Th.: Einführung in die transzendenten Zahlen. Springer, Berlin (1957) Zbl 0077.04703 MR 0086842 